\title{
Deformationen isolierter Kurvensingularitäten mit eingebetteten Komponenten
}

\author{
Christian Brücker, Gert-Martin Greuel
}

We study deformations of isolated curve singularities of arbitrary embedding dimension which may have embedded components. The existence of an embedded component has the effect that the generic fibre consists of isolated (fat) points and disjoint 1-dimensional components. We introduce for isolated curve singularities and for fat points a Milnor number $\mu$ and a $\delta$-invariant which generalize the well known invariants for reduced curve singularities. The invariants $\mu, \delta$ and the length of the embedded component control the topology of the generic fibre in a way which is surprisingly similar to the case of reduced curves, although many new phenomena occur.

Einleitung

\section{Inhalt}

1. Invarianten und induzierte Deformationen

2. Ungemischte und reduzierte Deformationen

3. Allgemeine Deformationen

4. Zusammenhangskomponenten der allgemeinen Faser

5. Globale projektive Kurven

6. Singularitäten der homologischen Dimension $\leq 2$

Literatur

\section{Einleitung}

Es ist ein bekanntes Phänomen, daß in Familien komplexer Räume spezielle Fasern eingebettete Komponenten besitzen, die für die Erklärung von Eigenschaften der allgemeinen Faser entscheidende Bedeutung haben. In der vorliegenden Arbeit wird in systematischer Weise der Einfluß von eingebetteten Komponenten einer isolierten komplexen Kurvensingularität auf die Nachbarfasern einer kleinen Deformation untersucht.

Als wichtigste Invarianten dienen dabei die Größe der nichtreduzierten Struktur $\varepsilon$ sowie die $\delta$-Invariante und die Milnorzahl $\mu$, die in dieser Arbeit 
für beliebige isolierte Kurvensingularitäten eingeführt wird und die bekannte Milnor-Zahl für reduzierte Kurvensingularitäten ([B-G]) verallgemeinert. Die Definition ist so gewählt, daß möglichst viele der bekannten topologischen Eigenschaften von $\mu$ erhalten bleiben. Notwendigerweise gehen dabei andere bekannte Eigenschaften von $\mu$, wie zum Beispiel die Halbstetigkeit bei Deformationen, verloren. Außerdem kann $\mu$ negativ werden.

Der wichtigste Unterschied zum reduzierten Fall ist der, daß bei kleinen Deformationen der Singularität $X$ die generische Faser $\mathcal{X}_{t}$ im Allgemeinen weder zusammenhängend noch reindimensional ist.

Für die Anzahl der null- respektive eindimensionalen Zusammenhangskomponenten geben wir in $\S 4$ (scharfe) Abschätzungen mit Hilfe der Invarianten $\varepsilon$, welche die „Größe" der nichtreduzierten Struktur mißt. Eine bessere Formel nur in Termen von $X$ kann es im Allgemeinen nicht geben, da die Zahl der Zusammenhangskomponenten von der speziellen Deformation abhängt.

In den $\S \S 2$ und 3 werden Zusammenhänge hergeleitet und bewiesen, die die bekannten Aussagen für den reduzierten Fall verallgemeinern. Hier zeigt sich, daß die neue Definition von $\mu$,richtig“ in dem Sinne ist, daß sie die algebraischen Ursachen für topologische Eigenschaften der deformierten Kurve erfaßt, welche für jede Deformation gelten.

In $§ 4$ wird schließlich gezeigt, daß die Anzahl der Zusammenhangskomponenten der allgemeinen Faser (im reduzierten Fall stets 1) und die Differenz der Milnorzahlen der speziellen und der allgemeinen Faser (im reduzierten Fall stets nichtnegativ) im nichtreduzierten Fall durch die $\varepsilon$-Invariante beschränkt sind. Weiter wird gezeigt, daß verschiedene Komponenten einer Singularität durch eine Deformation nur dann getrennt werden können, wenn $\varepsilon$ mindestens so groß ist wie die Schnittzahl dieser Komponenten.

In $\S 5$ untersuchen wir das Verhalten von Deformationen globaler projektiver Kurven.

In $\S 6$ werden abschließend Deformationen von 1-codimensionalen Varietäten der homologischen Dimension kleiner oder gleich 2 des $\mathbb{C}^{n}$ (hierunter fallen insbesondere alle Kurven in der Ebene mit eingebetteten Komponenten) untersucht.

Zur Theorie reduzierter Kurvensingularitäten sei auf [B-G] verwiesen, im Zusammenhang mit ,simultaner Normalisierung“ auch auf [Te]. Außerdem sei erwähnt, daß J. Steenbrink in einem Manuskript [St] ebenfalls Kurven mit eingebetteten Komponenten untersucht hat und für den Fall einer reinen Glättung (cf. 2.3.) analoge Resultate erzielte. Für die Überlassung seines Manuskriptes sowie für das folgende Beispiel danken wir ihm herzlich.

Notationen. Sei $X$ ein eindimensionaler komplexer Raum mit isolierten Singularitäten. Dann läßt sich $X$ schreiben als disjunkte Vereinigung $X=X^{0} \amalg X^{1}$, so daß $X^{0}$ nulldimensional und $X^{1}$ rein 1-dimensional mit isolierten Singularitäten ist.

Im Folgenden bezeichne $(X, 0) \subset\left(\mathbb{C}^{n}, 0\right)$ stets den Keim einer isolierten komplexen Kurvensingularität. 
Unter einem guten Repräsentanten $X$ von $(X, 0)$ verstehen wir den Durchschnitt eines beliebigen Repräsentanten von $(X, 0)$ mit einer Kugel $B=B_{\epsilon}(0) \subset$ $\mathbb{C}^{n}$ um den Ursprung des $\mathbb{C}^{n}$ mit hinreichend kleinem Radius $\epsilon$. Insbesondere wird $X-\{0\}$ grundsätzlich als glatt angenommen. Wir betrachten stets gute Repräsentanten.

Eine Deformation des Kurvenkeims $(X, 0)$ ist ein flacher Abbildungskeim $f:(\mathcal{X}, 0) \rightarrow(\mathbb{C}, 0)$, wo $(\mathcal{X}, 0)$ der Keim eines zweidimensionalen komplexen Raumes (nicht notwendig reindimensional) ist, so daß $\mathcal{O}_{X, 0} \cong \mathcal{O}_{\mathcal{X}, 0} \otimes_{\mathcal{O}_{\mathbb{\Phi}, 0}} \mathbb{C}$.

Unter einem guten Repräsentanten von $f$ verstehen wir eine Deformation $f: \mathcal{X} \rightarrow T$ eines guten Repräsentanten $X$ von $(X, 0)$, d.h. eine Abbildung $f: \mathcal{X} \rightarrow T$ mit folgenden Eigenschaften:

i) $\quad T$ ist eine kleine Kreisscheibe um 0 in $\mathbb{C}$ mit Radius $\eta \ll \epsilon$;

ii) $\quad \mathcal{X}$ ist eine abgeschlossene Untervarietät von $B \times T$;

iii) $\quad f$ ist die Einschränkung der Projektion $B \times T \rightarrow T$;

iv) $\quad f$ ist flach

v) $\quad f^{-1}(0) \cong X$

Wir schreiben im folgenden , $f: \mathcal{X} \rightarrow T$ ist eine Deformation von $X$ " für , $f$ ist ein guter Repräsentant der Deformation $f:(\mathcal{X}, 0) \rightarrow(\mathbb{C}, 0)^{“}$. Wir bezeichnen die Faser $f^{-1}(t)$ mit $\mathcal{X}_{t}$.

Für einen topologischen Raum $Y$ sei $b^{i}(Y)$ die $i$-te Bettizahl und $\chi(Y)$ die topologische Eulercharakteristik.

Beispiel (Steenbrink [St])

Sei $X \subset \mathbb{C}^{4}$ gegeben durch das Ideal $\mathrm{I}(X)=\left(x^{2}-y^{3}, x z, y z, w x, w y, w z, w^{2}\right)=$ $\left(x^{2}-y^{3}, z, w\right) \cap(x, y, w) \cap\left(x, y, z, w^{2}\right)$, d.h. $X$ ist die transversale Vereinigung einer Spitze in $\{0\} \times \mathbb{C}^{2}$ mit der Geraden $\mathbb{C} \times\{0\}$ und einer eingebetteten Komponente, die in die vierte Dimension zeigt.

Wir betrachten zwei verschiedene Deformationen dieser Kurve. 
Dabei ist die erste Deformation gegeben durch $\mathrm{I}(\mathcal{X})=\left(x^{2}-y^{3}+t^{2} y, x z, y z\right.$, $\left.w x, w y, w z-t z, w^{2}-t w\right)=\left(x^{2}-y^{3}+t^{2} y, z, w\right) \cap(x, y, w-t) \subset \mathbb{C}[t, w, x, y, z]$, die zweite durch $\mathrm{I}(\mathcal{X})=\left(x^{2}-y^{3}, x z-t y^{2}, y z-t w, w x-t y^{2}, w y-t x, w z-t^{3}\right.$, $\left.w^{2}-t^{2} y\right) \subset \mathbb{C}[t, w, x, y, z]$.

Es handelt sich also um zwei Glättungen $\mathcal{X}_{t}$ von $X$ mit verschiedner erster Bettizahl: Im ersten Fall gilt $b^{1}\left(\mathcal{X}_{t}\right)=2$, im zweiten Fall $b^{1}\left(\mathcal{X}_{t}\right)=1$. Man beachte aber, daß $b^{0}\left(\mathcal{X}_{t}\right)=2$ respektive $b^{0}\left(\mathcal{X}_{t}\right)=1$, d.h. die topologischen Eulercharakteristiken sind gleich.

\section{$\S 1$. Invarianten und induzierte Deformationen}

\subsection{Invarianten}

1.1.1. Sei $X$ ein (nicht notwendig reindimensionaler) 1-dimensionaler komplexer Raum mit isolierten Singularitäten. $\mathrm{E}_{X} \subset \mathcal{O}_{X}$ bezeichne die Idealgarbe der 0dimensionalen isolierten sowie der eingebetteten Komponenten, d.h. $\mathrm{E}_{X, x}=$ $\mathrm{H}_{\{x\}}^{0}\left(\mathcal{O}_{X}\right)$ ist das Ideal der auf $x$ konzentrierten Schnitte von $\mathcal{O}_{X}$. Der zu $X$ gehörige ungemischte Raum ist $X^{u}:=X_{\text {red }}^{1}$ mit der Strukturgarbe $\mathcal{O}_{X^{u}}=$ $\mathcal{O}_{X} / \mathrm{E}_{X}$. Unter der (ungemischten) Normalisierung von $X$ verstehen wir die Normalisierung von $X^{u}$ mit der kanonischen Abbildung $n: \overline{X^{u}} \rightarrow X^{u} \hookrightarrow X$. (Die Normalisierung einer reduzierten Kurve $Y$ wird stets mit $\bar{Y}$, die einer reduzierten Fläche $\mathcal{Y}$ dagegen mit $\tilde{\mathcal{Y}}$ bezeichnet.)

\section{Definition 1.1.2.}

$$
\begin{aligned}
& \varepsilon(X, x):=\operatorname{dim}_{\mathbb{T}} \mathrm{E}_{X, x} \quad \text { (E-Invariante) } \\
& \delta^{u}(X, x):=\operatorname{dim}_{\mathbb{T}}\left(n_{*} \mathcal{O}_{\overline{X^{u}}}\right)_{x} / \mathcal{O}_{X^{u}, x} \quad \text { (ungemischte } \delta \text {-Invariante) } \\
& \delta(X, x):=\delta^{u}(X, x)-\varepsilon(X, x) \quad \text { ( } \delta \text {-Invariante) } \\
& r(X, x):=\text { Anzahl der (eindimensionalem) irreduziblen Komponenten } \\
& \text { von }(X, x) \\
& r^{\prime}(X, x):=r(X, x)-1 \\
& \mu(X, x):=2 \delta(X, x)-r^{\prime}(X, x) \quad \text { (Milnorzahl) }
\end{aligned}
$$

Bemerkung 1.1.3. Es ist $\delta^{u} \geq 0$, und $\delta^{u}=0$ gilt genau dann, wenn $x \in X^{0}$ oder $\left(X_{\text {red }}^{1}, x\right)$ glatt ist. Insbesondere können sowohl $\delta$ als auch $\mu$ negativ sein. Ist $x$ ein isolierter Punkt von $X$, so gilt $\delta(X, x)=-\operatorname{dim}_{\mathbb{W}} \mathcal{O}_{X, x}$ und $\mu(X, x)=$ $2 \delta(X, x)+1$.

Wir bemerken, daß $-\delta(X, x)$ gerade die Eulercharakteristik $\chi\left(\mathcal{O}_{X, x}\right)=$ $\operatorname{dim}_{\mathbb{W}} \mathrm{H}^{0}\left(\mathcal{O}_{X, x}\right)-\operatorname{dim}_{\mathbb{T}} \mathrm{H}^{1}\left(\mathcal{O}_{X, x}\right)$ des Komplexes

$$
\mathcal{O}_{X, x} \quad: \quad 0 \rightarrow \mathcal{O}_{X, x}^{0}=\mathcal{O}_{X, x} \rightarrow \mathcal{O}_{X, x}^{1}=\left(n_{*} \mathcal{O}_{\overline{X^{u}}}\right)_{x} \rightarrow 0
$$

ist und daß $\overline{X^{u}}=\overline{X^{\text {red }}}$ gilt, wenn $X$ ein guter Repräsentant des Kurvenkeimes $(X, x)$ ist.

Ist $f: \mathcal{X} \rightarrow T$ ein guter Repräsentant einer Deformation von $(X, 0)$, so benutzen wir stets die Bezeichnung $\alpha_{t}=\alpha\left(\mathcal{X}_{t}\right)=\sum_{x \in \mathcal{X}_{t}} \alpha\left(\mathcal{X}_{t}, x\right)$, falls $\alpha$ eine 
der obigen Invarianten und diese Summe endlich ist (z.B. $\mu_{0}=\mu\left(\mathcal{X}_{0}, 0\right)=$ $\mu(X, 0))$.

\subsection{Induzierte Deformationen}

1.2.1. Sei $f: \mathcal{X} \rightarrow T$ eine fest vorgegebene Deformation eines guten Repräsentanten $X$ des Keimes $(X, 0)$. Dann bezeichne

$-\mathcal{X}^{\text {red }}$ die Reduktion von $\mathcal{X}, f^{\text {red }}: \mathcal{X}^{\text {red }} \rightarrow T$ die Einschränkung von $f$ (definiert durch das Bild von $f$ in $\mathcal{O}_{\mathcal{X}^{\text {red }}}$ ), $n: \tilde{\mathcal{X}}^{\text {red }} \rightarrow \mathcal{X}^{\text {red }}$ die Normalisierung, $\tilde{f}^{\text {red }}=f^{\text {red }} \circ n: \tilde{\mathcal{X}}^{\text {red }} \rightarrow T, \mathcal{X}_{t}^{\text {red }}=\left(f^{\text {red }}\right)^{-1}(t), \tilde{\mathcal{X}}_{t}^{\text {red }}=\left(\tilde{f}^{\text {red }}\right)^{-1}(t)$;

$-\mathcal{X}^{2}$ (resp. $\left.\mathcal{X}^{1}\right)$ den rein zwei-(resp. ein-)dimensionalen Anteil von $\mathcal{X}^{\text {red }}, f^{2}$ : $\mathcal{X}^{2} \rightarrow T$ die Einschränkung, $n: \tilde{\mathcal{X}}^{2} \rightarrow \mathcal{X}^{2}$ die Normalisierung, $\tilde{f}^{2}=f^{2} \circ n$ : $\tilde{\mathcal{X}}^{2} \rightarrow T, \mathcal{X}_{t}^{2}=\left(f^{2}\right)^{-1}(t), \tilde{\mathcal{X}}_{t}^{2}=\left(\tilde{f}^{2}\right)^{-1}(t)$

$-w: \hat{\mathcal{X}} \rightarrow \mathcal{X}^{2}$ die schwache Normalisierung (oder Maximalisierung, vgl. [Fi]) von $\mathcal{X}^{2}, \hat{f}=f^{2} \circ w: \hat{\mathcal{X}} \rightarrow T, \hat{\mathcal{X}}_{t}=\hat{f}^{-1}(t)$;

$-\mathcal{X}^{*}$ die die disjunkte Vereinigung derjenigen 2-dimensionalen abgeschlossenen Unterräume $\mathcal{X}_{i}$ von $\mathcal{X}^{2}$, die sich nur in 0 treffen $\left(\mathcal{X}_{i}\right.$ sind die Abschlüsse in $\mathcal{X}^{2}$ der Zusammenhangskomponenten von $\left.\mathcal{X}^{2}-\{0\}\right), \pi: \mathcal{X}^{*} \rightarrow \mathcal{X}^{2}$ die kanonische Projektion, $f^{*}=f^{2} \circ \pi: \mathcal{X}^{*} \rightarrow T$ und $\mathcal{X}_{t}^{*}=\left(f^{*}\right)^{-1}(t)$.

Proposition 1.2.2. Die Abbildungs(multi)keime $f^{\mathrm{red}}, \tilde{f^{\mathrm{red}}}, f^{2}, \tilde{f}^{2}, \hat{f}$ und $f^{*}$ sind flach.

Damit sind $f^{\text {red }}, \tilde{f}^{\text {red }}, f^{2}, \tilde{f}^{2}, \hat{f}$ und $f^{*}$ (gegebenenfalls nach Verkleinerung des Repräsentanten $f: \mathcal{X} \rightarrow T$ ) gute Repräsentanten von Deformationen der speziellen Faser. Man beachte, daß diese spezielle Faser i.A. von der Deformation $f$ und nicht nur von $X$ abhängt. Alle induzierten Deformationen werden später bei der Untersuchung der Fasern $\mathcal{X}_{t}$ von $f: \mathcal{X} \rightarrow T$ benötigt.

Beweis: Die zu betrachtenden Abbildungskeime sind die Bilder von $f$ in den entsprechenden (semi-)lokalen Ringen, die wie folgt zusammenhängen:

$$
\begin{array}{ccc} 
& \left(n_{*} \mathcal{O}_{\tilde{\mathcal{X}}^{\mathrm{red}}}\right)_{0} & \left(n_{*} \mathcal{O}_{\tilde{\mathcal{X}}^{2}}\right)_{0} \\
\mathcal{O}_{\mathcal{X}^{2}, 0} \oplus \mathcal{O}_{\mathcal{X}^{1}, 0} & \left(\pi_{*} \mathcal{O}_{\mathcal{X}^{*}}\right)_{0} & \left(w_{*} \mathcal{O}_{\hat{\mathcal{X}}}\right)_{0} . \\
\mathcal{O}_{\mathcal{X}, 0} & \mathcal{O}_{\mathcal{X}^{2}, 0}
\end{array}
$$

Man zeigt direkt, daß $f^{\text {red }}$ ein Nichtnullteiler in $\mathcal{O}_{\mathcal{X}^{\text {red }, 0}}$ ist. Nach dem folgenden Lemma ist damit das Bild von $f^{\text {red }}$ in $\mathcal{O}_{\mathcal{X}^{2}, 0} \oplus \mathcal{O}_{\mathcal{X}^{1}, 0}$ ein Nichtnullteiler und daher auch $f^{2}$ in $\mathcal{O}_{\mathcal{X}^{2}, 0}$. Die restlichen Aussagen folgen aus

Lemma 1.2.3. Sei $(\mathcal{X}, 0)$ ein zweidimensionaler reduzierter Raumkeim, $f$ : $(\mathcal{X}, 0) \rightarrow(\mathbb{C}, 0)$ flach und $\pi:\left(\mathcal{X}^{\prime}, 0^{\prime}\right) \rightarrow(\mathcal{X}, 0)$ eine partielle Normalisierung 
(i.e. $\left(\mathcal{X}^{\prime}, 0^{\prime}\right)$ ist $($ Multi-) $)$ keim mit $\mathcal{O}_{\mathcal{X}, 0} \subset\left(\pi_{*} \mathcal{O}_{\mathcal{X}^{\prime}}\right)_{0} \subset\left(n_{*} \mathcal{O}_{\tilde{\mathcal{X}}}\right)_{0}$ und $n: \tilde{\mathcal{X}} \rightarrow \mathcal{X}$ die Normalisierung von $\mathcal{X})$. Dann ist $f \circ \pi:\left(\mathcal{X}^{\prime}, 0\right) \rightarrow(\mathbb{C}, 0)$ flach.

Beweis: $\quad$ Für $\mathcal{X}^{\prime}=\tilde{\mathcal{X}}$ findet man einen Beweis z.B. in [B-G], Theorem 4.1.4(1). Also ist $f \circ n$ Nichtnullteiler in $\left(n_{*} \mathcal{O}_{\tilde{\mathcal{X}}}\right)_{0}$ und damit $f \circ \pi$ in $\left(\pi_{*} \mathcal{O}_{\mathcal{X}^{\prime}}\right)_{0}$.

Lemma 1.2.4. Für die speziellen Fasern $\mathcal{X}_{0}^{2}$ bzw. $\mathcal{X}_{0}^{*}$ von $f^{2}$ bzw. $f^{*}$ gilt: $\delta\left(\mathcal{X}_{0}^{2}\right)=\delta\left(\mathcal{X}_{0}^{*}\right)$

Beweis: Ohne Einschränkung der Allgemeinheit sei $\mathcal{X}=\mathcal{X}^{2}$ und $\mathcal{X}_{0}^{2}=X$. Es ist $-\delta(X)$ die Eulercharakteristik des Komplexes $\mathcal{O}_{X, 0}$ und entsprechend ist $-\delta\left(\mathcal{X}_{0}^{*}\right)$ die Eulercharacteristik von $\mathcal{O}_{\mathcal{X}_{0}^{*}, 0^{*}}=\bigoplus_{x \in 0^{*}} \mathcal{O}_{\mathcal{X}_{0}^{*}, x}, 0^{*}=\pi^{-1}(0)$, wobei für einen Multikeim $\delta$ gleich der Summe der $\delta$-Invarianten der einzelnen Keime ist.

Die Abbildung $\mathcal{O}_{\mathcal{X}, 0} \hookrightarrow\left(\pi_{*} \mathcal{O}_{\mathcal{X}^{*}}\right)_{0}$ induziert eine Abbildung $\mathcal{O}_{X, 0} \rightarrow \mathcal{O}_{\mathcal{X}_{0}^{*}, 0^{*}}$ und damit eine Abbildung der Komplexe $\mathcal{O}_{X, 0} \rightarrow \mathcal{O}_{\mathcal{X}_{0}^{*}, 0^{*}}$. Da $X_{\text {red }}$ und $\mathcal{X}_{0, \text { red }}^{*}$ dieselbe Normalisierung haben, ist diese Abbildung außer im Grad 0 ein Isomorphismus. Im Grad 0 haben wir die exakte Sequenz

$$
0 \rightarrow K e r \rightarrow \mathcal{O}_{X, 0} \rightarrow \mathcal{O}_{\mathcal{X}_{0}^{*}, 0^{*}} \rightarrow K o k \rightarrow 0,
$$

und $\delta(X)-\delta\left(\mathcal{X}_{0}^{*}\right)=\chi\left(\mathcal{O}_{\mathcal{X}_{0}^{*}, 0^{*}}\right)-\chi\left(\mathcal{O}_{X, 0}\right)=\operatorname{dim}_{\mathbb{T}} K o k-\operatorname{dim}_{\mathbb{W}} K e r$.

Aus dem Schlangenlemma zu

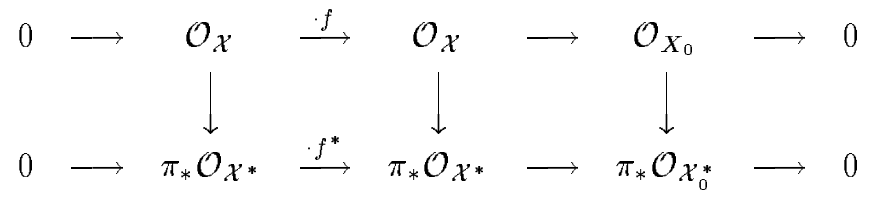

und der Tatsache, daß $\pi_{*} \mathcal{O}_{\mathcal{X}^{*}} / \mathcal{O}_{\mathcal{X}}$ auf 0 konzentriert ist, folgt $\operatorname{dim}_{\mathbb{W}} K e r=$ $\operatorname{dim}_{\mathbb{C}} K o k$ und damit die Behauptung.

\subsection{Der reduzierte Fall}

Ist $(X, 0)$ eine reduzierte Kurvensingularität, so sind die obigen Definitionen den üblichen, in $[\mathrm{B}-\mathrm{G}]$ und $[\mathrm{Gr}]$ verwendeten Definitionen äquivalent, und es gilt (vgl. [B-G],4.2.2,4.2.4,5.2.2):

Proposition 1.3.1. Sei $X$ ein guter Repräsentant von $(X, 0)$ und $f: \mathcal{X} \rightarrow T$ eine Deformation von $X$, dann gilt:

a) Für alle $t$ ist

(i) $b_{t}^{0}=1$, d.h. $\mathcal{X}_{t}$ ist zusammenhängend,

(ii) $\mu_{0}-\mu_{t}=b_{t}^{1}$,

(iii) $\mu_{0}-\mu_{t} \geq \delta_{0}-\delta_{t} \geq 0$.

b) Folgende Bedingungen sind äquivalent:

(i) $\mu_{t}$ ist konstant für alle $t$, 
(ii) $\delta_{t}$ und $r_{t}^{\prime}$ sind konstant für alle $t$,

(iii) $b_{t}^{1}=0$ für alle $t$,

(iv) $\mathcal{X}_{t}$ ist zusammenziehbar für alle $t$.

Eine äquivalente Formulierung für a) (ii) ist, $\mu_{0}-\mu_{t}=1-\chi_{t}$ ", während sich b) (i) bzw. b) (ii) wie folgt formulieren lassen: , $\mu_{t}-b_{t}^{0}$ ist konstant für alle $t^{\text {", }}$ bzw. , $\delta_{t}$ und $r_{t}^{\prime}+b_{t}^{0}$ sind konstant für alle $t^{\prime \prime}$. In dieser Form werden wir den Satz verwenden und verallgemeinern.

\section{§. Ungemischte und reduzierte Deformationen}

Wir betrachten zunächst nur Deformationen $f: \mathcal{X} \rightarrow T$ von $X$, für die der Totalraum $\mathcal{X}$ reduziert bzw. ungemischt (reduziert und rein 2-dimensional) ist. Solche Deformationen nennen wir reduziert bzw. ungemischt. Wir werden später sehen, daß dies genau dann gilt, wenn die allgemeine Faser $\mathcal{X}_{t}, t \neq 0$, reduziert bzw. ungemischt (reduziert und rein 1-dimensional) ist (vgl. Proposition 3.1.1). Die spezielle Faser dagegen kann eingebettete Komponenten haben.

Wir erinnern daran, daß dann $n: \tilde{\mathcal{X}} \rightarrow \mathcal{X}$ die Normalisierung, $\tilde{f}=f \circ n$, $\tilde{\mathcal{X}}_{t}=\tilde{f}^{-1}(t)$ und $\tilde{0}=n^{-1}(0)$ ist.

Den meisten Untersuchungen dieses Paragraphen liegt folgendes exakte Di$\operatorname{agramm}(\Delta)$ von $\mathcal{O}_{\mathcal{X}}$-Moduln zugrunde:

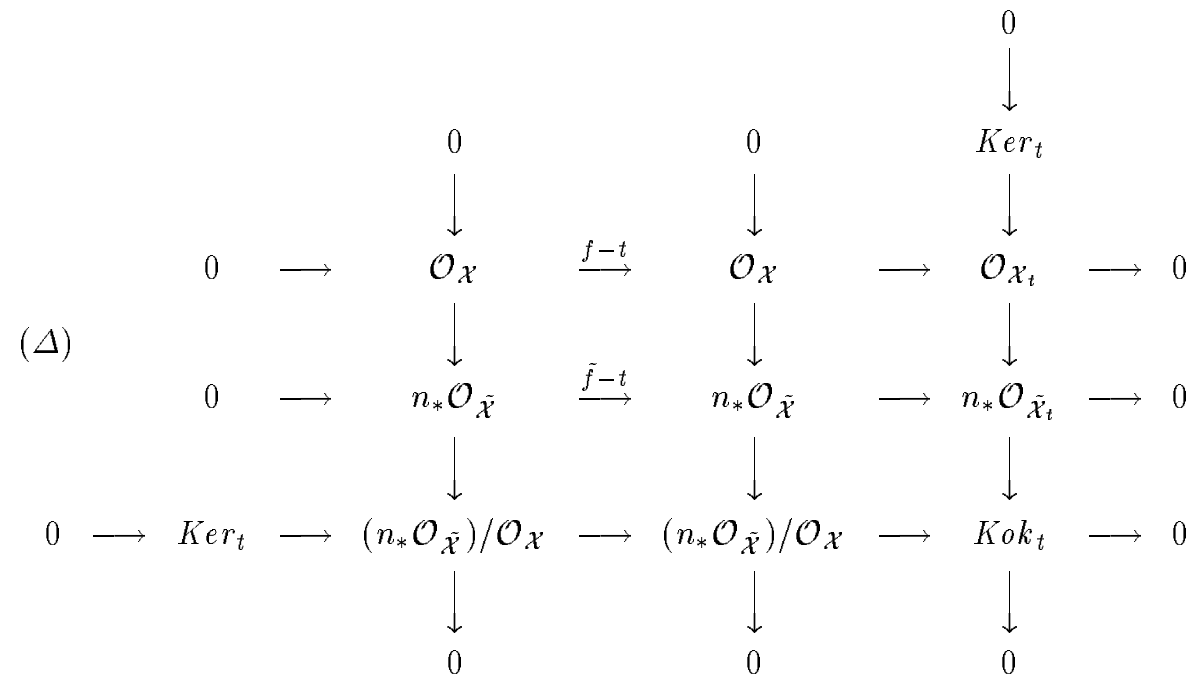

Es ist $f_{*}\left(\left(n_{*} \mathcal{O}_{\tilde{\mathcal{X}}}\right) / \mathcal{O}_{\mathcal{X}}\right)$ als $\mathcal{O}_{T}$-Modul eine direkte Summe $\mathcal{F} \oplus \mathcal{T}$, wo $\mathcal{F}$ frei über $\mathcal{O}_{T}$ und $\mathcal{T}=\mathrm{H}_{\{0\}}^{0}\left(f_{*}\left(\left(\pi_{*} \mathcal{O}_{\mathcal{X}^{*}}\right) / \mathcal{O}_{\mathcal{X}}\right)\right)$, die Torsion, ein endlichdimensionaler $\mathbb{C}$-Vektorraum ist. 


\subsection{Die Normalisierung der Deformation}

Lemma 2.1.1. Für eine ungemischte Deformation $f: \mathcal{X} \rightarrow T$ von $X$ gilt:

(1) $\tilde{\mathcal{X}}_{0}$ ist ungemischt,

(2) $\tilde{\mathcal{X}}_{t}$ ist glatt und ungemischt für $t \neq 0$.

Ist $f$ nur reduziert, so ist $\tilde{\mathcal{X}}_{t}$ noch glatt für $t \neq 0$.

Beweis:

(1) $\tilde{\mathcal{X}}$ ist normal, also hat $\mathcal{O}_{\tilde{\mathcal{X}}}$ die Tiefe 2 ([Ma], S. 124). Damit hat $\mathcal{O}_{\tilde{\mathcal{X}}_{0}}$ die Tiefe 1, d.h. $\tilde{\mathcal{X}}_{0}$ ist Cohen-Macaulay'sch. Da nun $\tilde{\mathcal{X}}_{0}-\tilde{0} \cong X-\{0\}$, ist $\tilde{\mathcal{X}}_{0}$ höchstens in $\tilde{0}$ nicht reduziert. Da andererseits $\mathcal{O}_{\tilde{\mathcal{X}}_{0}} \mathrm{C}-\mathrm{M}$ ist, ist $\mathrm{H}_{\tilde{0}}^{0}\left(\mathcal{O}_{\tilde{\mathcal{X}}_{0}}\right)=0$, also $\tilde{\mathcal{X}}_{0}$ reduziert.

(2) $\tilde{f}$ ist flach, also ist $\operatorname{Sing}(\tilde{f})=\left\{x \in \tilde{\mathcal{X}} \mid x \in \operatorname{Sing}\left(\tilde{\mathcal{X}}_{\tilde{f}(x)}\right)\right\}$. $\tilde{\mathcal{X}}$ ist normal, also ist (gegebenenfalls nach geeigneter Einschränkung) $\tilde{\mathcal{X}}-\tilde{0}$ glatt. Deshalb ist $\operatorname{Sing}(\tilde{f})-\tilde{0}=\left\{x \in \tilde{\mathcal{X}}-\tilde{0} \mid \frac{\partial \tilde{f}}{\partial x_{i}}(x)=0 \quad \forall i\right\}$, wo $x_{i}$ lokale Koordinaten von $\tilde{\mathcal{X}}$ in $x$ sind, und damit ist $\tilde{f} \mid \operatorname{Sing}(\tilde{f})$ lokal konstant. Da aber jede Komponente von $\operatorname{Sing}(\tilde{f})$ auch einen Punkt $x$ von $\tilde{0}$ enthält (T hinreichend klein), ist $\tilde{f} \mid \operatorname{Sing}(\tilde{f})=$ 0 . Damit ist aber $\tilde{\mathcal{X}}_{t}$ glatt für $t \neq 0$ und rein 1-dimensional, falls $f$ ungemischt ist.

$\tilde{f}$ ist also die Glättung einer reduzierten Kurve. Ferner ist wegen der universellen Eigenschaft der Normalisierung, $\tilde{\mathcal{X}}_{t}=\overline{\left(\mathcal{X}_{t}\right)}$ die Normalisierung von $\mathcal{X}_{t}$.

\subsection{Die allgemeine Faser}

Proposition 2.2.1. Sei $f: \mathcal{X} \rightarrow T$ eine ungemischte bzw. reduzierte Deformation. Dann ist $\mathcal{X}_{t}$ ungemischt bzw. reduziert für $t \neq 0$.

Beweis: Offensichtlich ist $\mathcal{X}_{t}$ reindimensional, falls $f$ ungemischt ist.

Betrachte nun das Diagramm $(\Delta)$. Wegen $\mathcal{T}=0$ für $t \neq 0$ ist $K e r_{t}=0$. Da $\tilde{\mathcal{X}}_{t}$ glatt ist, ist $\mathcal{O}_{\tilde{\mathcal{X}}_{t}}$ reduziert und damit auch $\mathcal{O}_{\mathcal{X}_{t}}$.

\subsection{Invarianten der allgemeinen Faser}

Lemma 2.3.1. Seif $: \mathcal{X} \rightarrow T$ eine reduzierte Deformation von $X, \mathcal{X}=\mathcal{X}^{2} \cup \mathcal{X}^{1}$ die Zerlegung in den rein $2-$ und den rein 1-dimensionalen Anteil von $\mathcal{X}$ sowie Ker und Kok der Kern bzw. der Kokern der Abbildung $\mathcal{O}_{X, 0} \rightarrow \mathcal{O}_{\tilde{\mathcal{X}}_{0}, 0}$, dann gilt:

$$
\operatorname{dim}_{\mathbb{C}} K o k-\operatorname{dim}_{\mathbb{C}} K e r=\delta_{t}+\varepsilon_{t} \text { für } t \neq 0,
$$


wobei $\varepsilon_{t}=\varepsilon\left(\tilde{\mathcal{X}}_{0}\right)=\#\left(f \mid \mathcal{X}^{1}\right)^{-1}(t)$ die Anzahl der isolierten Punkte von $\mathcal{X}_{t}$ ist. Speziell ist $\varepsilon_{t}=0$ für ungemischtes $f$.

Beweis: Wir berechnen $\operatorname{dim}_{\mathbb{C}} K o k-\operatorname{dim}_{\mathbb{C}} K e r$ nach der untersten Zeile des Diagramms $(\Delta)$ für $t=0$. Die Zerlegung der mittleren Terme in einen freien Teil $\mathcal{F}$ und einen Torsionsteil zeigt $\operatorname{dim}_{\mathbb{C}} K o k-\operatorname{dim}_{\mathbb{T}} K e r=\operatorname{dim}_{\mathbb{T}} \mathcal{F} / f \mathcal{F}=$ $\operatorname{dim}_{\mathbb{C}} n_{*} \mathcal{O}_{\tilde{\mathcal{X}}_{t}} / \mathcal{O}_{\mathcal{X}_{t}}$, da $\mathcal{F}$ ein freier $\mathcal{O}_{T}$-Modul ist, der für $t \neq 0$ mit $n_{*} \mathcal{O}_{\tilde{\mathcal{X}}} / \mathcal{O}_{\mathcal{X}}$ übereinstimmt. Nun ist $\tilde{\mathcal{X}}_{t}$ glatt nach Lemma 2.1.1 und $\mathcal{X}_{t}$ reduziert nach Proposition 2.2.1, also ist $\tilde{\mathcal{X}}_{t}$ die Normalisierung von $\mathcal{X}_{t}$ und die rechte Seite gleich $\delta_{t}+\varepsilon_{t}$. Da $\tilde{\mathcal{X}}_{0}$ nach Lemma 2.1.1 höchstens in den isolierten Punkten nicht reduziert ist, folgt $\varepsilon_{t}=\varepsilon\left(\tilde{\mathcal{X}}_{t}\right)=\varepsilon\left(\tilde{\mathcal{X}}_{0}\right)$.

Lemma 2.3.2. Ist $f: \mathcal{X} \rightarrow T$ ungemischt, so gilt mit obigen Bezeichnungen $\operatorname{dim}_{\mathbb{T}} \operatorname{Ker}=\varepsilon_{0}$.

Beweis: $\quad$ Da $\tilde{\mathcal{X}}_{0}$ reduziert ist, ist $\operatorname{Ker}=\mathrm{H}_{\{0\}}^{0}\left(\mathcal{O}_{X}\right)=\mathrm{E}_{0}$.

Satz 2.3.3. Ist $f: \mathcal{X} \rightarrow T$ eine ungemischte Deformation von $X$, dann gilt:

(1) $\delta_{0}-\delta_{t}=\delta\left(\tilde{\mathcal{X}}_{0}\right)$ für $t \neq 0$,

(2) $\mu_{0}-\mu_{t}=1-\chi_{t}$ für alle $t$.

Bemerkung. Wir werden diesen Satz später auf beliebige Deformationen verallgemeinern (vgl. 3.1.2).

Beweis:

(1) Es ist $\delta_{0}-\delta\left(\tilde{\mathcal{X}}_{0}\right)=-\chi\left(\mathcal{O}_{X, 0}\right)+\chi\left(\mathcal{O}_{\tilde{\mathcal{X}}_{0}, \tilde{0}}\right)=\operatorname{dim}_{\mathbb{C}} K e r-\operatorname{dim}_{\mathbb{C}} K o k=\delta_{t}$ nach Lemma 2.3.1.

(2) Mittels Mayer-Vietoris folgt $\chi_{t}=\chi\left(\mathcal{X}_{t}\right)=\chi\left(\tilde{\mathcal{X}}_{t}\right)-r_{t}^{\prime}$. Da $\tilde{\mathcal{X}}_{0}$ reduziert und $\tilde{f}$ eine Glättung ist, folgt aus dem reduzierten Fall (1.3) $2 \delta\left(\tilde{\mathcal{X}}_{0}\right)-r^{\prime}\left(\tilde{\mathcal{X}}_{0}\right)=\mu\left(\tilde{\mathcal{X}}_{0}\right)=$ $s-\chi\left(\tilde{\mathcal{X}}_{t}\right)$, wobei $s=\# \tilde{0}$ die Anzahl der Zusammenhangskomponenten von $\tilde{\mathcal{X}}_{t}$ ist. Aus $r^{\prime}\left(\tilde{\mathcal{X}}_{0}\right)+s=r_{0}$ und aus (1) folgt dann $\chi\left(\mathcal{X}_{t}\right)=-2\left(\delta_{0}-\delta_{t}\right)+r_{0}-r_{t}^{\prime}=$ $-\mu_{0}+1+\mu_{t}$.

Definition 2.3.4. Sei $f: \mathcal{X} \rightarrow T$ eine Deformation von $X$.

1) $f$ heißt $\delta$-konstant, wenn $\delta_{t}=\delta_{0}$ für alle $t$.

2) $\quad f$ heißt Glättung bzw. reine Glättung von $X$, wenn $\mathcal{X}_{t}$ glatt bzw. reindimensional und glatt ist für $t \neq 0$.

Insbesondere sind reine Glättungen stets ungemischte Deformationen.

Es ergeben sich leicht folgende Folgerungen:

Korollar 2.3.5. Eine ungemischte Deformation $f: \mathcal{X} \rightarrow T$ ist genau dann $\delta$ konstant, wenn die Normalisierung $n: \tilde{\mathcal{X}} \rightarrow \mathcal{X}$ von $\mathcal{X}$ alle Fasern $\mathcal{X}_{t}$ simultan normalisiert (d.h. wenn $\tilde{\mathcal{X}}_{t}$ für alle $t$ glatt ist). 
Beweis: Da $\tilde{\mathcal{X}}_{0}$ reduziert und rein 1-dimensional ist, gilt $\delta\left(\tilde{\mathcal{X}}_{0}\right)=0$ genau dann, wenn $\tilde{\mathcal{X}}_{0}$ glatt ist.

Proposition 2.3.6. Ist $f: \mathcal{X} \rightarrow T$ eine reine Glättung von $X$, dann gilt:

(i) $\delta(X) \geq 0$

(ii) $\chi_{t}=1-\mu_{0}$ für $t \neq 0$. Insbesondere hängt $\chi_{t}$ nicht von der reinen Glättung $f a b$.

Korollar 2.3.7. Ist $\delta(X)<0$, so ist $X$ nicht rein glättbar.

\subsection{Verschwindende Zykel}

Für Deformationen reduzierter Kurven gilt stets $b_{t}^{1}=\mu_{0}-\mu_{t} \geq \delta_{0}-\delta_{t} \geq 0$ für alle $t$.

Im nichtreduzierten Fall ist diese Aussage nur dann richtig, wenn die allgemeine Faser zusammenhängend ist.

Proposition 2.4.1. Sei $f: \mathcal{X} \rightarrow T$ eine ungemischte Deformation von $X$. Dann ist die Anzahl der Zusammenhangskomponenten von $\mathcal{X}_{t}$ für $t \neq 0$ gleich der Anzahl der Zusammenhangskomponenten von $\mathcal{X}-\{0\}$.

Beweis:

(a) Sei $\mathcal{X}$ irreduzibel. Dann ist $\mathcal{X}-\{0\}$ sowie $\tilde{\mathcal{X}}$ und damit $\tilde{\mathcal{X}}_{0}$ zusammenhängend. Da $\tilde{\mathcal{X}}_{0}$ reduziert ist, ist dann $\tilde{\mathcal{X}}_{t}$ für $t \neq 0$ zusammenhängend ([B$\mathrm{G}], 4.2 .2)$. Da die Normalisierung stetig ist, ist also auch $\mathcal{X}_{t}$ zusammenhängend für $t \neq 0$.

(b) Sei $\mathcal{X}$ reduzibel. Dann ist $\mathcal{X}=\bigcup_{i=1}^{s} \mathcal{X}^{i}$, wo die $\mathcal{X}^{i}$ die irreduziblen Komponenten sind. Mit $f^{i}:=f \mid \mathcal{X}^{i}$ und $\mathcal{X}_{t}^{i}:=\left(f^{i}\right)^{-1}(t)$ ist dann $\mathcal{X}_{t}=\bigcup_{i=1}^{s} \mathcal{X}_{t}^{i}$. Sei $\mathcal{X}^{i, j}:=\mathcal{X}^{i} \cap \mathcal{X}^{j}, \mathcal{X}_{t}^{i, j}:=\mathcal{X}_{t}^{i} \cap \mathcal{X}_{t}^{j}$ für $i \neq j$. Mit $f^{i, j}:=f \mid \mathcal{X}^{i, j}$ ist dann offensichtlich $\mathcal{X}_{t}^{i, j}=\left(f^{i, j}\right)^{-1}(t)$. Offenbar ist für alle $i \neq j, 0 \in \mathcal{X}^{i, j}$.

Da nun $X$ in $x \in X-\{0\}$ glatt ist, ist auch $\mathcal{X}$ glatt in $x$. Insbesondere ist $\mathcal{X}$ lokal irreduzibel in $x$, das heißt, $x$ liegt nicht im Durchschnitt zweier irreduzibler Komponenten . Deshalb ist $\mathcal{X}^{i, j} \cap X=\{0\} \quad \forall i \neq j$.

Die Behauptung folgt dann aus der Tatsache, daß offensichtlich $\mathcal{X}^{i, j}$ genau dann von $\{0\}$ verschieden ist, wenn $\mathcal{X}^{i, j}$ eine Kurve, also $f^{i, j}: \mathcal{X}^{i, j} \rightarrow T$ surjektiv und deshalb $\mathcal{X}_{t}^{i, j}=\left(f^{i, j}\right)^{-1}(t)$ nicht leer ist.

Proposition 2.4.2. Sei $f: \mathcal{X} \rightarrow T$ eine ungemischte Deformation von $X$ und sei $\mathcal{X}_{t}$ zusammenhängend für $t \neq 0$. Dann ist $\mu_{0}-\mu_{t}=b_{t}^{1} \geq \delta_{0}-\delta_{t} \geq 0$ für alle $t$.

Beweis: Sei $t \neq 0$. Da $\delta_{0}-\delta_{t}=\delta\left(\tilde{\mathcal{X}}_{0}\right) \geq 0$, (da $\tilde{\mathcal{X}}_{0}$ reduziert), ist nur noch zu zeigen, daß $\mu_{0}-\mu_{t}=b_{t}^{1} \geq \delta_{0}-\delta_{t}$ gilt. 
Da $\mathcal{X}_{t}$ zusammenhängend ist, ist $b_{t}^{0}=1$, also $\chi_{t}=1-b_{t}^{1}$ für $t \neq 0$. Mit Satz $2.3 .3(2)$ folgt $\mu_{0}-\mu_{t}=b_{t}^{1}$.

Es bleibt zu zeigen, daß $\mu_{0}-\mu_{t} \geq \delta_{0}-\delta_{t}$. Dies folgt aus Lemma 2.4.4

Satz 2.4.3. Ist $f: \mathcal{X} \rightarrow T$ eine ungemischte Deformation von $X$, dann gilt $b_{t}^{1} \geq \delta_{0}-\delta_{t} \geq 0$ für alle $t$.

Beweis: Betrachte die induzierte Deformation $f^{*}: \mathcal{X}^{*} \rightarrow T$. Nach Konstruktion ist $\mathcal{X}^{*}=\bigsqcup \mathcal{X}_{i}^{*}$, wo die $\mathcal{X}_{i}^{*}-\{0\}$ genau die Zusammenhangskomponenten von $\mathcal{X}-\{0\}$ sind, und es ist $\mathcal{X}_{t}^{*}=\mathcal{X}_{t}$ für $t \neq 0$. Folglich gilt $\delta_{t}=\delta\left(\mathcal{X}_{t}^{*}\right)$ und nach Lemma 1.2.4 gilt auch $\delta_{0}=\delta\left(\mathcal{X}_{0}^{*}\right)$. Da sowohl $b^{1}$ wie auch $\delta$ additiv bei disjunkter Vereinigung sind, folgt die Behauptung aus Proposition 2.4.2.

Um den Beweis von Proposition 2.4.2 zu beenden, betrachten wir die induzierte Deformation $\hat{f}: \hat{\mathcal{X}} \rightarrow T$. Da die schwache Normalisierung zusammenhängend ist, ist auch die spezielle Faser $\hat{\mathcal{X}}_{0}$ zusammenhängend und es ist $r^{\prime}\left(\hat{\mathcal{X}}_{0}\right)=r^{\prime}(X) . \hat{\mathcal{X}}_{0}$ braucht nicht schwach normal zu sein, aber $X_{\text {red }}$ und $\hat{\mathcal{X}}_{0, \text { red }}$ haben die gleiche schwache Normalisierung $\hat{X}_{\text {red }}$.

Lemma 2.4.4. Seif $: \mathcal{X} \rightarrow T$ eine ungemischte Deformation von $X$, dann gilt $\mathcal{O}_{\hat{\mathcal{X}}_{0}} \subset \mathcal{O}_{\hat{X}_{\text {red }}}$ und es ist

$$
\left(\mu_{0}-\delta_{0}\right)-\left(\mu_{t}-\delta_{t}\right)=\operatorname{dim}_{\mathbb{C}} \mathcal{O}_{\hat{X}_{\text {red }}} / \mathcal{O}_{\hat{\mathcal{X}}_{0}} \geq 0
$$

Speziell ist $f$ genau dann eine $(\mu-\delta)$-konstante Deformation, wenn $\hat{f}: \hat{\mathcal{X}} \rightarrow T$ simultane schwache Normalisierung ist (d.h. $\hat{\mathcal{X}}_{t}$ ist für alle $t$ schwach normal).

Bemerkung. Eine reduzierte Kurvensingularität $(X, 0)$ mit $r$ Zweigen ist genau dann schwach normal, wenn $(X, 0)$ isomorph zum $r$-Achsenkreuz in $\left(\mathbb{C}^{r}, 0\right)$ ist. Folglich gilt $\delta(X, 0)-r^{\prime}(X, 0)=\operatorname{dim}_{\mathbb{C}} \mathcal{O}_{\hat{X}, o} / \mathcal{O}_{X, 0}$.

Beweis des Lemmas: Betrachte das Diagramm $(\Delta)$, in dem $n_{*} \mathcal{O}_{\tilde{\mathcal{X}}}$ durch $w_{*} \mathcal{O}_{\hat{\mathcal{X}}}$ ersetzt sei. Seien Ker und Kok der Kern bzw. der Kokern der Abbildung $\mathcal{O}_{X} \rightarrow$ $\mathcal{O}_{\hat{\mathcal{X}}_{0}}$. Analog zu Lemma 2.3.1 zeigt man $\operatorname{dim}_{\mathbb{C}} K o k-\operatorname{dim}_{\mathbb{C}} K e r=\delta_{t}-r_{t}^{\prime}$.

$\hat{\mathcal{X}}$ hat wie $\tilde{\mathcal{X}}$ die Tiefe 2 und daher folgt wie in 2.1, daß $\hat{\mathcal{X}}_{0}$ reduziert ist. Also ist $\operatorname{dim}_{\mathbb{C}} \operatorname{Ker}=\varepsilon_{0}$ und $\mathcal{O}_{\hat{\mathcal{X}}_{0}} \rightarrow \mathcal{O}_{\hat{X}_{\text {red }}}$ injektiv. Betrachte nun die Inklusionen $\mathcal{O}_{X_{\text {red }}} \hookrightarrow \mathcal{O}_{\hat{X}_{0}} \hookrightarrow \mathcal{O}_{\hat{X}_{\text {red }}}$.

Es folgt $\operatorname{dim}_{\mathbb{T}} K o k=\left(\delta_{0}-r_{0}^{\prime}+\varepsilon_{0}\right)-\operatorname{dim}_{\mathbb{C}} \mathcal{O}_{\hat{X}_{\text {red }}} / \mathcal{O}_{\hat{X}_{0}}$. Berücksichtigt man noch $\mu-\delta=\delta-r^{\prime}$, so folgt die Behauptung.

\section{§3. Allgemeine Deformationen}

In diesem Paragraphen werden wir die gewünschten Aussagen über die Deformation $f: \mathcal{X} \rightarrow T$ unter Benutzung der Ergebnisse des vorigen Paragraphen im wesentlichen auf Aussagen über $f^{2}: \mathcal{X}^{2} \rightarrow T$ zurückführen.

Da nämlich nach Konstruktion $f^{2}$ eine ungemischte Deformation von $\mathcal{X}_{0}^{2}$ ist, gelten für $f^{2}$ die Aussagen von $\S 2$. Insbesondere gilt: 
$\mathcal{X}_{t}^{2}$ ist ungemischt für $t \neq 0$ (Proposition 2.2.1), $\tilde{\mathcal{X}}_{0}^{2}$ ist reduziert und $\tilde{\mathcal{X}}_{t}^{2}$ ist glatt für $t \neq 0$ (Lemma 2.1.1), $\delta\left(\tilde{\mathcal{X}}_{0}^{2}\right)=\delta\left(\mathcal{X}_{0}^{2}\right)-\delta\left(\mathcal{X}_{t}^{2}\right)$ für $t \neq 0$ und $\mu\left(\mathcal{X}_{0}^{2}\right)-$ $\mu\left(\mathcal{X}_{t}^{2}\right)=1-\chi\left(\mathcal{X}_{t}^{2}\right)$ für alle $t$ (Satz 2.3.3), $b^{1}\left(\mathcal{X}_{t}^{2}\right) \geq \delta\left(\mathcal{X}_{0}^{2}\right)-\delta\left(\mathcal{X}_{t}^{2}\right) \geq 0$ für alle $t$ (Satz 2.4.3).

\subsection{Der nulldimensionale Anteil der allgemeinen Faser}

Proposition 3.1.1.

(1) Für jede Deformation $f: \mathcal{X} \rightarrow T$ ist $\varepsilon\left(\mathcal{X}_{0}^{2}\right)=\varepsilon_{0}-\varepsilon_{t}$ für $t \neq 0$.

(2) $f$ ist ungemischt $\Leftrightarrow \mathcal{X}_{t}$ ist ungemischt für $t \neq 0 \Leftrightarrow \varepsilon_{t}=0$ für $t \neq 0$.

(3) $f$ ist reduziert $\Leftrightarrow \mathcal{X}_{t}$ ist reduziert für $t \neq 0 \Leftrightarrow \varepsilon_{t}=\#\{$ isolierte Punkte von $\left.\mathcal{X}_{t}\right\}$ für $t \neq 0$.

Beweis: Betrachte folgendes exakte Diagramm von $\mathcal{O}_{\mathcal{X}}$-Moduln:

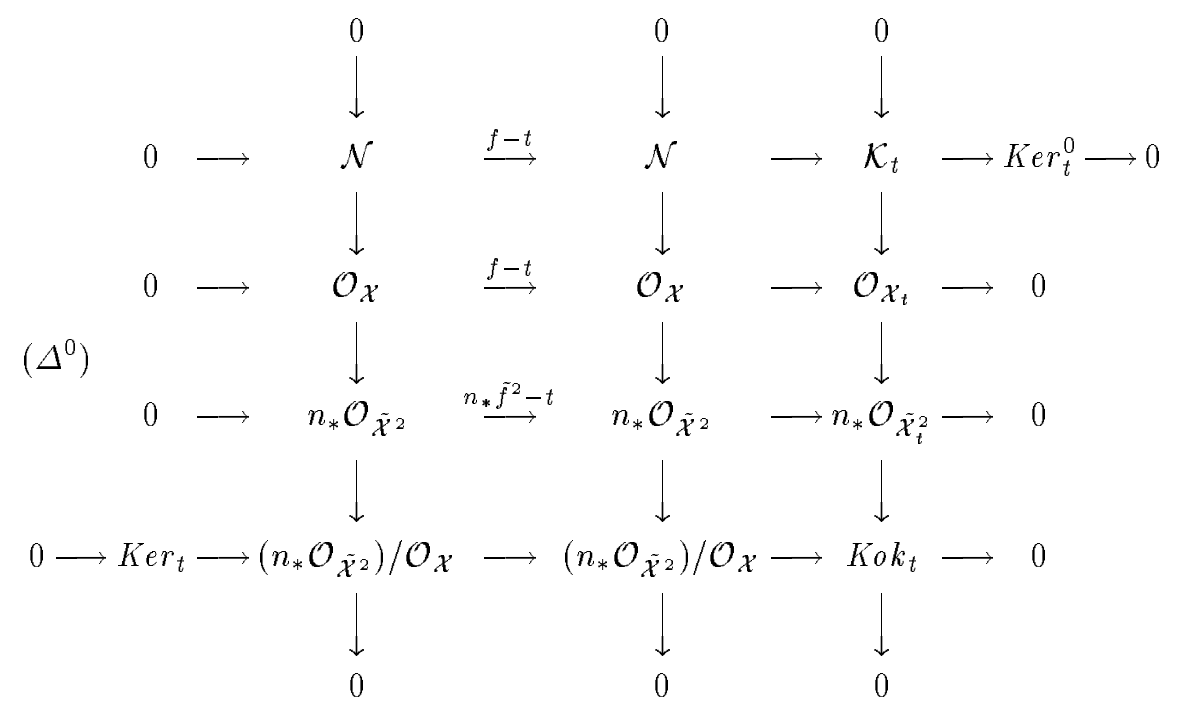

Betrachte $\left(\Delta^{0}\right)$ zunächst für $t=0$. Nach dem Schlangenlemma ist $K e r_{0}^{0} \cong K e r_{0}$. Da $\mathcal{O}_{\mathcal{X}}$ und $\mathcal{O}_{\mathcal{X}^{2}}$ dasselbe Bild in $n_{*} \mathcal{O}_{\tilde{\mathcal{X}}^{2}}$ haben, ist nach Lemma 2.3.2 Ker $_{0}=$ $\mathrm{E}_{\mathcal{X}_{0}^{2}}$. Da $\tilde{\mathcal{X}}_{0}^{2}$ reduziert ist, folgt analog $\mathcal{K}=\mathrm{E}_{0}$. Nach der ersten Zeile von $\left(\Delta^{0}\right)$ ist $\varepsilon\left(\mathcal{X}_{0}^{2}\right)=\varepsilon_{0}-\operatorname{dim}_{\mathbb{T}} \mathcal{N} / f \mathcal{N}$. Zu zeigen: $\operatorname{dim}_{\mathbb{W}} \mathcal{N} / f \mathcal{N}=\varepsilon_{t}$ für $t \neq 0$.

Betrachte nun das Diagramm $\left(\Delta^{0}\right)$ für $t \neq 0$ : Da $\mathrm{E}\left(\tilde{\mathcal{X}}_{t}^{2}\right)=0$, ist $\varepsilon_{t}=$ $\operatorname{dim}_{\mathbb{C}} \mathcal{N} /(f-t) \mathcal{N}$.

Da $\mathcal{N}$ als Untermodul von $\mathcal{O}_{\mathcal{X}}$ flach über $\mathcal{O}_{T}$ ist und als $\mathcal{O}_{T}$-Modul endlich erzeugt, ist er frei über $\mathcal{O}_{T}$. Also ist $\varepsilon_{t}=\operatorname{dim}_{\mathbb{T}} \mathcal{N} / f \mathcal{N}$ für $t \neq 0$. Das beweist (1).

Die Richtungen $\Rightarrow$ für (2) und (3) wurden schon in Proposition 2.2.1 und in Lemma 2.3.1 gezeigt. 
Ist $\varepsilon_{t}=0$ für $t \neq 0$, so ist $\mathcal{N}=0$, da $\mathcal{N}$ frei ist. Also ist $\mathcal{O}_{\mathcal{X}}=\mathcal{O}_{\mathcal{X}^{2}}$ und $\mathcal{X}$ ungemischt.

Ist $\varepsilon_{t}$ gleich der Anzahl der isolierten Punkte von $\mathcal{X}_{t}$ für $t \neq 0$, dann ist $\mathcal{N}$ auf $\mathcal{X}^{1}$ konzentriert, also ist $\mathcal{X}$ in $\mathcal{X}^{2}-\{0\}$ reduziert. Für $x \in \mathcal{X}^{1}-\{0\}$ gilt $\operatorname{dim}_{\mathbb{C}} \mathcal{N}_{x} / f \mathcal{N}_{x}=1$, also ist $\mathcal{X}$ auch in $\mathcal{X}^{1}-\{0\}$ reduziert. Da $f \in \mathcal{O}_{\mathcal{X}, 0}$ Nichtnullteiler ist, kann $\mathcal{X}$ keine in 0 konzentrierte eingebettete Komponente haben, also ist $\mathcal{X}$ reduziert.

Mit Hilfe von Proposition 3.1.1 lassen sich nun viele Aussagen über beliebige Deformationen $f$ auf solche über die Deformation $f^{2}$ zurückführen. Insbesondere gilt der folgende

Satz 3.1.2. Für jede Deformation $f: \mathcal{X} \rightarrow T$ gilt:

(i) $\delta\left(\tilde{\mathcal{X}}_{0}^{2}\right)=\delta_{0}-\delta_{t}$ für $t \neq 0$,

(ii) $\mu_{0}-\mu_{t}=1-\chi_{t}$ für alle $t$,

(iii) $b_{t}^{1} \geq \delta_{0}-\delta_{t} \geq 0$ für alle $t$.

Beweis: Für $t \neq 0$ ist $\delta_{0}-\delta_{t}=\delta^{u}(X)-\varepsilon_{0}-\delta^{u}\left(\mathcal{X}_{t}\right)+\varepsilon_{t}=\delta^{u}\left(\mathcal{X}_{0}^{2}\right)-\delta^{u}\left(\mathcal{X}_{t}^{2}\right)-$ $\varepsilon\left(\mathcal{X}_{0}^{2}\right)$ (Prop. 3.1.1) $=\delta^{u}\left(\mathcal{X}_{0}^{2}\right)-\varepsilon\left(\mathcal{X}_{0}^{2}\right)-\delta\left(\mathcal{X}_{t}^{2}\right)\left(\right.$ da $\mathcal{X}_{t}^{2}$ ungemischt $)=\delta\left(\mathcal{X}_{0}^{2}\right)-$ $\delta\left(\mathcal{X}_{t}^{2}\right)$.

Ferner gilt für beliebige eindimensionale komplexe Räume $Y=Y^{1} \cup Y^{0}\left(Y^{i}=\right.$ rein $i$-dimensionaler Teil) mit isolierten Singularitäten:

$$
\begin{aligned}
& r^{\prime}(Y)=r^{\prime}\left(Y^{0}\right)+r^{\prime}\left(Y^{1}\right), \text { wo } r^{\prime}\left(Y^{1}\right)=r^{\prime}\left(Y^{u}\right) \text { und } r^{\prime}\left(Y^{0}\right)=-b^{0}\left(Y^{0}\right), \\
& \chi(Y)=\chi\left(Y^{\text {red }}\right)=\chi\left(Y^{u}\right)+\chi\left(Y^{0, \text { red }}\right)=\chi\left(Y^{u}\right)+b^{0}\left(Y^{0}\right),
\end{aligned}
$$

also $r^{\prime}(Y)=r^{\prime}\left(Y^{u}\right)-b^{0}\left(Y^{0}\right), \chi(Y)=\chi\left(Y^{u}\right)+b^{0}\left(Y^{0}\right)$.

(i) $\delta_{0}-\delta_{t}=\delta\left(\mathcal{X}_{0}^{2}\right)-\delta\left(\mathcal{X}_{t}^{2}\right)=\delta\left(\tilde{\mathcal{X}}_{0}^{2}\right)$ für $t \neq 0$.

(ii) $\mu_{0}-\mu_{t}=2 \delta_{0}-r_{0}^{\prime}-2 \delta_{t}+r_{t}^{\prime}=2\left(\delta_{0}-\delta_{t}\right)-r^{\prime}(X)+r^{\prime}\left(\mathcal{X}_{t}\right)=2\left(\delta\left(\mathcal{X}_{0}^{2}\right)-\delta\left(\mathcal{X}_{t}^{2}\right)\right)-$ $r^{\prime}\left(\mathcal{X}_{0}^{2}\right)+r^{\prime}\left(\mathcal{X}_{t}^{2}\right)-b^{0}\left(\left(\mathcal{X}_{t}\right)^{0}\right)=2 \delta\left(\mathcal{X}_{0}^{2}\right)-r^{\prime}\left(\mathcal{X}_{0}^{2}\right)-2 \delta\left(\mathcal{X}_{t}^{2}\right)+r^{\prime}\left(\mathcal{X}_{t}^{2}\right)-b^{0}\left(\left(\mathcal{X}_{t}\right)^{0}\right)=$ $\mu\left(\mathcal{X}_{0}^{2}\right)-\mu\left(\mathcal{X}_{t}^{2}\right)-b^{0}\left(\left(\mathcal{X}_{t}\right)^{0}\right)=1-\chi\left(\mathcal{X}_{t}^{2}\right)-b^{0}\left(\left(\mathcal{X}_{t}\right)^{0}\right)=1-\left(\chi\left(\mathcal{X}_{t}^{2}\right)+b^{0}\left(\left(\mathcal{X}_{t}\right)^{0}\right)=\right.$ $1-\chi_{t}$.

(iii) Da $b^{1}\left(Y^{0}\right)=0$, ist $b^{1}\left(\mathcal{X}_{t}\right)=b^{1}\left(\left(\mathcal{X}_{t}\right)^{1}\right)=b^{1}\left(\mathcal{X}_{t}^{2}\right) \geq \delta\left(\mathcal{X}_{0}^{2}\right)-\delta\left(\mathcal{X}_{t}^{2}\right)=$ $\delta_{0}-\delta_{t} \geq 0$.

\subsection{Folgerungen}

Korollar 3.2.1. Eine Deformation $f: \mathcal{X} \rightarrow T$ ist genau dann $\delta$-konstant, wenn die Abbildung $n: \tilde{\mathcal{X}}^{2} \rightarrow \mathcal{X}$ alle Fasern $\mathcal{X}_{t}$ simultan normalisiert (d.h. wenn $\tilde{\mathcal{X}}_{t}^{2}$ glatt für alle $t$ ).

Beweis: Satz 3.1.2 und Korollar 2.3.5.

Korollar 3.2.2. Für beliebige Deformationen $f: \mathcal{X} \rightarrow T$ von $X$ ist $\mu_{0}-\mu_{t} \geq$ $1-b_{t}^{0}$. 
Beweis: Satz 3.1.2(ii).

Satz 3.2.3. Folgende Bedingungen sind äquivalent:

(i) $\mu_{t}-b_{t}^{0}$ ist konstant für alle $t$,

(ii) $\delta_{t}$ und $r_{t}^{\prime}+b_{t}^{0}$ sind konstant für alle $t$,

(iii) $b_{t}^{1}=0$ für alle $t$.

Beweis: $\quad($ i $) \Leftrightarrow($ iii $)$ : Nach Satz 3.1.2(ii) ist $\mu_{0}-\mu_{t}=1-\chi_{t}=1-b_{t}^{0}+b_{t}^{1}$, also $b_{t}^{1}=\left(\mu_{0}-1\right)-\left(\mu_{t}-b_{t}^{0}\right)$.

(ii) $\Rightarrow($ i $): \mu_{t}-b_{t}^{0}=2 \delta_{t}-r_{t}^{\prime}-b_{t}^{0}=2 \delta_{0}-r_{0}^{\prime}-1=\mu_{0}-1$.

(i), (iii) $\Rightarrow$ (ii): Nach Satz 3.1.2(iii) ist $0=b_{t}^{1} \geq \delta_{0}-\delta_{t} \geq 0$, also ist $\delta_{0}=\delta_{t}$, und deshalb ist $r_{t}^{\prime}+b_{t}^{0}=2 \delta_{t}-\mu_{t}+b_{t}^{0}=2 \delta_{0}-\mu_{0}+1=r_{0}^{\prime}+1$.

\section{§4. Zusammenhangskomponenten der allgemeinen Faser}

Für Deformationen reduzierter Kurven gilt stets $b_{t}^{0}=1$ für alle $t$. Wie gesehen, ist dies für Deformationen nichtreduzierter Kurven im allgemeinen nicht richtig. Somit stellt sich die Frage nach einer allgemeinen, nur von der Singularität $(X, 0)$ abhängigen Abschätzung für $b_{t}^{0}$. Dazu benötigen wir einen allgemeinen Begriff einer Schnittzahl.

\subsection{Schnittzahl}

\section{Definition 4.1.1.}

(i) Seien $(X, 0),(Y, 0) \subset\left(\mathbb{C}^{n}, 0\right)$ beliebige Kurvenkeime mit Idealen $\mathrm{I}(X)$, $\mathrm{I}(Y) \subset \mathcal{O}_{\mathbb{C}^{n}, 0}$. Wir setzen

$$
(X . Y)_{0}:=s_{0}(\{X, Y\}):=\operatorname{dim}_{\mathbb{C}} \mathcal{O}_{\mathbb{C}^{n}, 0} /(\mathrm{I}(X)+\mathrm{I}(Y))
$$

und nennen dies die Schnittzahl von $X$ und $Y$ in 0 .

(ii) Für endlich viele Kurvenkeime $\left(X_{i}, 0\right) \subset\left(\mathbb{C}^{n}, 0\right), i=1, \ldots, p$ mit Idealen $\mathrm{I}\left(X_{i}\right)$ sei $\left(X^{(k)}, 0\right)$ definiert durch $\mathrm{I}\left(X^{k}\right):=\bigcap_{i=1}^{k} \mathrm{I}\left(X_{i}\right)$.

Wir nennen

$$
s_{0}\left(\left\{X_{i}\right\}_{i=1, \ldots, p}\right):=\sum_{k=1}^{p-1}\left(X^{(k)} \cdot X_{k+1}\right)_{0}
$$

die Schnittzahl der $X_{i}, i=1, \ldots, p$ in 0 .

Bemerkung. $s_{0}\left(\left\{X_{i}\right\}\right)$ ist unabhängig von der Einbettung und ist offensichtlich genau dann endlich, wenn für $i \neq j$ die Keime $\left(X_{i}, 0\right)$ und $\left(X_{j}, 0\right)$ keine gemeinsamen (nicht eingebetteten) Komponenten haben. Das folgende Lemma verallgemeinert die in [B-G] für reduzierte Kurvensingularitäten bewiesene Aussage. 
Lemma 4.1.2. Seien $\left(X_{1}, 0\right), \ldots,\left(X_{p}, 0\right) \subset\left(\mathbb{C}^{n}, 0\right)$ Kurvenkeime mit isolierter Singularität, so daß für $i \neq 0\left(X_{i}, 0\right)$ und $\left(X_{j}, 0\right)$ keine gemeinsamen Komponenten haben. Dann gilt

(i) $s_{0}\left(\left\{X_{i}\right\}_{i=1, \ldots, p}\right)=\delta\left(X^{(p)}, 0\right)-\sum_{i=1}^{p} \delta\left(X_{i}, 0\right)$,

(ii) $s_{0}\left(\left\{X_{i}\right\}_{i=1, \ldots, p}\right)-s_{0}\left(\left\{X_{i}^{u}\right\}_{i=1, \ldots, p}\right)=\sum_{i=1}^{p} \varepsilon\left(X_{i}, 0\right)-\varepsilon\left(X^{(p)}, 0\right) \geq 0$. Insbesondere hängt $s_{0}\left(\left\{X_{i}\right\}_{i=1, \ldots, p}\right)$ nicht von der Numerierung ab.

Beweis: Der Beweis erfolgt durch Induktion über $p$ (vgl.[B-G],S.9 für den reduzierten Fall).

a) Der Fall $p=2$. In diesem Fall ist $s_{0}\left(X_{1}, X_{2}\right)=\left(X_{1} \cdot X_{2}\right)_{0}$. Sei im folgenden $\mathcal{O}:=\mathcal{O}_{\mathbb{d}^{n}, 0} ; \mathrm{I}_{i}:=\mathrm{I}\left(X_{i}\right)$, $\mathrm{I}:=\mathrm{I}_{1} \cap \mathrm{I}_{2}=\mathrm{I}\left(X^{(2)}\right)$. Beachte, daß $\mathrm{I}_{i}^{u}:=\mathrm{I}\left(X_{i}^{u}\right)=\operatorname{rad}\left(\mathrm{I}_{i}\right)$ und $\mathrm{I}^{u}:=\mathrm{I}_{1}^{u} \cap \mathrm{I}_{2}^{u}=\operatorname{rad}(\mathrm{I})=\mathrm{I}\left(X^{(2), u}\right)$.

Da nun für beliebige Ideale $\mathbf{J}_{1}$ und $\mathbf{J}_{2}$ in $\mathcal{O}$ die Sequenz

$(*): 0 \rightarrow \mathcal{O} /\left(\mathrm{J}_{1} \cap \mathrm{J}_{2}\right) \stackrel{\varphi}{\rightarrow} \mathcal{O} / \mathrm{J}_{1} \oplus \mathcal{O} / \mathrm{J}_{2} \stackrel{\psi}{\rightarrow} \mathcal{O} /\left(\mathrm{J}_{1}+\mathrm{J}_{2}\right) \rightarrow 0$ exakt ist, betrachte folgendes exakte Diagramm:

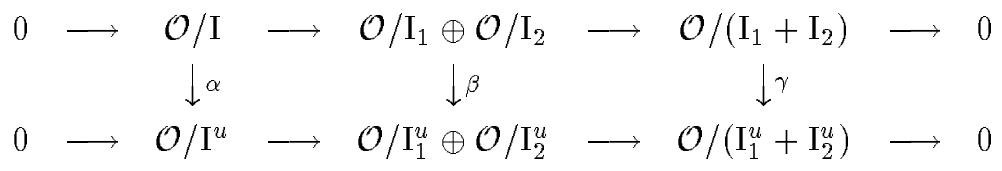

Da $\alpha$ und $\beta$ offensichtlich surjektiv sind, ist auch $\gamma$ surjektiv, und es ergibt sich die exakte Kern-Sequenz:

$$
0 \longrightarrow \mathrm{E}\left(X^{(2)}\right) \longrightarrow \mathrm{E}\left(X_{1}\right) \oplus \mathrm{E}\left(X_{2}\right) \longrightarrow K \longrightarrow 0
$$

Es folgt, daß $\varepsilon\left(X_{1}, 0\right)+\varepsilon\left(X_{2}, 0\right)-\varepsilon\left(X^{(2)}, 0\right)=\operatorname{dim}_{\mathbb{C}} K=\left(X_{1} \cdot X_{2}\right)_{0}-\left(X_{1}^{u} \cdot X_{2}^{u}\right)_{0}$. Dies ist Behauptung (ii).

$\mathrm{Zu}$ (i) bemerken wir, daß nach [B-G] $\delta^{u}\left(X^{(2)}, 0\right)=\delta\left(X_{1}^{u}, 0\right)+\delta\left(X_{2}, 0\right)+$ $\left(X_{1}^{u} \cdot X_{2}^{u}\right)_{0}$ gilt. Damit ist $\left(X_{1} \cdot X_{2}\right)_{0}=\left(X_{1}^{u} \cdot X_{2}^{u}\right)_{0}+\varepsilon\left(X_{1}, 0\right)+\varepsilon\left(X_{2}, 0\right)-\varepsilon\left(X^{(2)}, 0\right)=$ $\delta^{u}\left(X^{(2)}, 0\right)-\varepsilon\left(X^{(2)}, 0\right)-\delta\left(X_{1}^{u}, 0\right)+\varepsilon\left(X_{1}, 0\right)-\delta\left(X_{2}^{u}, 0\right)+\varepsilon\left(X_{2}, 0\right)=\delta\left(X^{(2)}, 0\right)-$ $\delta\left(X_{1}, 0\right)-\delta\left(X_{2}, 0\right)$.

b) Zum Schluß von $p$ auf $p+1$ setzen wir $X^{(p+1)}=X^{(p)} \cup X_{p+1}$. Die Behauptung folgt dann aus der Induktionsvoraussetzung und aus a).

4.1.3. Um die Zusammenhangskomponenten der allgemeinen Faser von $f$ : $\mathcal{X} \rightarrow T$ mit der Schnittzahl zu verbinden, betrachten wir die induzierte Deformation $f^{*}: \mathcal{X}^{*} \rightarrow T$. Sei also $\mathcal{X}^{2}=\bigcup_{i=1}^{p} \mathcal{X}_{i}, \mathcal{X}_{i} \cap \mathcal{X}_{j}=\{0\}$ für $i \neq j$ und $\mathcal{X}_{i}-\{0\}$ zusammenhängend. Dann ist $\mathcal{X}^{*}=\coprod_{i=1}^{p} \mathcal{X}_{i}$. Sei $\left(\mathcal{X}^{2}, 0\right) \subset\left(\mathbb{C}^{n}, 0\right)$ definiert durch das Ideal $\mathrm{I} \subset \mathcal{O}_{\mathbb{C}^{n}, 0}$, seien $\mathrm{I}_{i}$ die Ideale der $\mathcal{X}_{i}, \mathrm{I}=\bigcap_{i=1}^{p} \mathrm{I}_{i}$, und bezeichne $f \in \mathcal{O}_{\mathbb{T}^{n}, 0}$ auch ein Urbild von $f^{2} \in \mathcal{O}_{\mathcal{X}^{2}, 0}=\mathcal{O}_{\mathbb{T}^{n}, 0} /$ I. Dann ist $\mathcal{X}_{0}^{*}=\left(f^{*}\right)^{-1}(0)=\coprod_{i=1}^{p} X_{i}$ mit $X_{i}=\left(f^{*} \mid \mathcal{X}_{i}\right)^{-1}(0), \mathcal{O}_{X_{i}, 0}=\mathcal{O}_{\mathbb{C}^{n}, 0} / \mathrm{I}_{i}+(f)$, und $\mathcal{X}_{0}^{2}=\left(f^{2}\right)^{-1}(0), \mathcal{O}_{\mathcal{X}_{0}^{2}, 0}=\mathcal{O}_{\mathbb{C}^{n}, 0} / \mathrm{I}+(f)$. Wir setzen

$$
X^{\prime}:=\bigcup_{i=1}^{p} X_{i}, \quad \mathcal{O}_{X^{\prime}, 0}=\mathcal{O}_{\mathbb{C}^{n}, 0} / \bigcap_{i=1}^{p}\left(\mathrm{I}_{i}+(f)\right) .
$$


Beachte, daß i.A. $\mathrm{I}+(f) \varsubsetneqq \bigcap\left(\mathrm{I}_{i}+(f)\right)$, so daß $X^{\prime}$ ein echter Unterraum von $\mathcal{X}_{0}^{2}$ ist, der die gleiche Reduktion wie $\mathcal{X}_{0}^{2}$ hat, so daß $X^{u}=X^{\prime u}=\mathcal{X}_{0}^{2, u}=\bigcup_{i=1}^{p} \mathcal{X}_{i}^{u}$ mit $\mathcal{O}_{X_{i}^{u}, 0}=\mathcal{O}_{\mathbb{C}^{n}, o} / \operatorname{rad}\left(\mathrm{I}_{i}+(f)\right)$ gilt. Weiterhin ist $X_{i}^{u}$ i.A. nicht irreduzibel (es ist $p$ gleich der Anzahl der 1-dimensionalen Zusammenhangskomponenten der allgemeinen Faser $\mathcal{X}_{t}$ und damit i.A. kleiner als die Anzahl $r$ der Zweige von $(X, 0))$.

Proposition 4.1.4. Sei $f: \mathcal{X} \rightarrow T$ eine Deformation von $X$, dann gilt mit den oben eingeführten Bezeichnungen:

$\varepsilon_{0}-\varepsilon_{t}=\varepsilon\left(\mathcal{X}_{0}^{*}, 0\right)+s_{0}\left(\left\{X_{i}^{u}\right\}\right)=\varepsilon\left(X^{\prime}\right)+s_{0}\left(\left\{X_{i}\right\}\right)$.

Beweis: $\quad s_{0}\left(\left\{X_{i}^{u}\right\}\right)=\delta\left(X^{\prime u}, 0\right)-\sum_{i} \delta\left(X_{i}^{u}, 0\right)=\delta\left(\mathcal{X}_{0}^{2}\right)+\varepsilon\left(\mathcal{X}_{0}^{2}\right)-\sum_{i} \delta\left(X_{i}, 0\right)-$ $\sum_{i} \varepsilon\left(X_{i}, 0\right)$. Da $\sum_{i} \delta\left(X_{i}, 0\right)=\delta\left(\mathcal{X}_{0}^{*}\right)=\delta\left(\mathcal{X}_{0}^{2}\right)$ (nach Lemma 1.2.3) und $\sum_{i} \varepsilon\left(X_{i}, 0\right)=\varepsilon\left(\mathcal{X}_{0}^{*}\right)$, ist $s_{0}\left(\left\{X_{i}^{u}\right\}\right)=\varepsilon\left(\mathcal{X}_{0}^{2}\right)-\varepsilon\left(\mathcal{X}_{0}^{*}\right)=\varepsilon_{0}-\varepsilon_{t}-\varepsilon\left(\mathcal{X}_{0}^{*}\right)$ (Prop. 3.1.1).

Nach 4.1.2 folgt dann

$s_{0}\left(\left\{X_{i}\right\}\right)=\varepsilon_{0}-\varepsilon_{t}-\varepsilon\left(\mathcal{X}_{0}^{*}\right)+\sum_{i} \varepsilon\left(X_{i}, 0\right)-\varepsilon\left(X^{\prime}\right)=\varepsilon_{0}-\varepsilon_{t}-\varepsilon\left(X^{\prime}\right)$.

Korollar 4.1.5. $\varepsilon_{0}-\varepsilon_{t} \geq s_{0}\left(\left\{X_{i}^{u}\right\}\right)$ und Gleichheit gilt genau dann, wenn alle $X_{i}$ reduziert sind. Speziell ist $\varepsilon$ halbstetig und es ist $\varepsilon_{0}=\varepsilon_{t}$ für alle $t$ genau dann wenn der 1-dimensionale Teil $\left(\mathcal{X}_{t}\right)^{1}$ von $\mathcal{X}_{t}$ zusammenhängend ist und $\varepsilon\left(\mathcal{X}_{0}^{*}\right)=0$.

Bemerkung. Die Ungleichung kann man so lesen: Ist $X_{\text {red }}=X^{\prime} \cup X^{\prime \prime}$ mit $X^{\prime} \cap$ $X^{\prime \prime}=\{0\}$, dann lassen sich $X^{\prime}$ und $X^{\prime \prime}$ höchstens dann durch eine Deformation trennen, wenn $\varepsilon(X, 0) \geq\left(X^{\prime} . X^{\prime \prime}\right)_{0}$.

Satz 4.1.6. Für jede Deformation von $X$ gilt für $t \neq 0$

$b_{t}^{0} \leq \varepsilon_{t}+s_{0}\left(\left\{X_{i}^{u}\right\}\right)+1 \leq \varepsilon_{0}+1$.

Beweis: Sei $\mathcal{X}_{t}=\left(\mathcal{X}_{t}\right)^{0} \amalg\left(\mathcal{X}_{t}\right)^{1}$ die Zerlegung von $\mathcal{X}_{t}$ in den 0- und den 1dimensionalen Anteil, dann ist $b_{t}^{0}=b^{0}\left(\left(\mathcal{X}_{t}\right)^{0}\right)+b^{0}\left(\left(\mathcal{X}_{t}\right)^{1}\right)$. Wir haben $\varepsilon_{t}=$ $\varepsilon\left(\left(\mathcal{X}_{t}\right)^{0}\right)+\varepsilon\left(\left(\mathcal{X}_{t}\right)^{1}\right) \geq \varepsilon\left(\left(\mathcal{X}_{t}\right)^{0}\right) \geq b^{0}\left(\left(\mathcal{X}_{t}\right)^{0}\right)$ sowie $b^{0}\left(\left(\mathcal{X}_{t}\right)^{1}\right)=b^{0}\left(\mathcal{X}_{t}^{2}\right)=b^{0}\left(\mathcal{X}_{t}^{*}\right)=$ $b^{0}\left(\mathcal{X}_{0}^{*}\right)=: p$ und $X^{u}=\bigcup_{i=1}^{p} X_{i}^{u}$ wie oben. Es folgt $s_{0}\left(\left\{X_{i}^{u}\right\}_{i=1, \ldots, p}\right) \geq p-1$ und damit $b_{t}^{0}=b^{0}\left(X_{t}^{0}\right)+b^{0}\left(X_{t}^{1}\right) \leq \varepsilon_{t}+s_{0}\left(\left\{X_{i}^{u}\right\}\right)+1 \leq \varepsilon_{0}+1$.

Korollar 4.1.7. $\mu_{0}-\mu_{t} \geq b_{t}^{1}-\varepsilon_{0}$ für $t \neq 0$.

Beweis: $\quad \mu_{0}-\mu_{t}=1-\chi_{t}=1-b_{t}^{0}+b_{t}^{1} \geq b_{t}^{1}-\varepsilon_{0}$ 


\subsection{Eine Deformation mit maximalem $b_{t}^{0}$}

Nach Satz 4.1.6 ist $b_{t}^{0}$ stets kleiner oder gleich $\varepsilon_{0}+1$. Der Beweis liefert auch ein notwendiges und hinreichendes Kriterium, wann dieser Maximalwert tatsächlich angenommen wird. Wir geben ein Beispiel dafür, das gleichzeitig zeigt, daß alle möglichen Kombinationen von 0- und 1-dimensionalen Anteilen auftreten.

Satz. Seien $k$ und $q$ nichtnegative ganze Zahlen. Dann gibt es eine Kurve $X$ und eine Deformation $f: \mathcal{X} \rightarrow T$ von $X$ derart, daß $\varepsilon_{0}=k+q, b^{0}\left(\left(\mathcal{X}_{t}\right)^{0}\right)=k$ und $b^{0}\left(\left(\mathcal{X}_{t}\right)^{1}\right)=q+1$ für $t \neq 0$, insbesondere also $b_{t}^{0}=k+q+1=\varepsilon_{0}+1$ für $t \neq 0$.

Zum Beweis wird die gesuchte Deformation angegeben.

Sei $X_{0} \subset \mathbb{C}^{2 q+k+1}$ (mit den Koordinaten $x_{0}, \ldots, x_{q}, y_{1}, \ldots, y_{q}, z_{1}, \ldots, z_{k}$ ) gegeben durch das von der folgenden Menge $\mathcal{M}$ von Funktionen in $\mathcal{O}_{\mathbb{T}^{2 q+k+1}}$ erzeugte Ideal $\mathrm{I}\left(X_{0}\right)$ :

$$
\begin{aligned}
& \mathcal{M}:=\left\{x_{i} x_{j}\right\}_{0 \leq i<j \leq q} \cup\left\{x_{i} y_{j}\right\}_{0 \leq i \leq q, 1 \leq j \leq q} \cup\left\{x_{i} z_{j}\right\}_{0 \leq i \leq q, 1 \leq j \leq k} \\
& \cup\left\{y_{i} y_{j}\right\}_{1 \leq i \leq j \leq q} \cup\left\{y_{i} z_{j}\right\}_{1 \leq i \leq q, 1 \leq i \leq k} \cup\left\{z_{i} z_{j}\right\}_{1 \leq i \leq j \leq k},
\end{aligned}
$$

d.h., $\mathrm{I}(X)$ wird erzeugt von allen Monomen vom Grad 2 mit Ausnahme der $x_{i}^{2}$. Sei ferner $\mathcal{X} \subset \mathbb{C}^{2 q+k+2}$ (mit zusätzlicher Koordinate $t$ ) gegeben durch das von der folgenden Menge $\mathcal{M}^{\prime}$ von Funktionen in $\mathcal{O}_{\mathbb{C}^{2 q+k+2}}$ erzeugte Ideal $\mathrm{I}(\mathcal{X})$ :

$$
\begin{aligned}
\mathcal{M}^{\prime}:= & \left\{x_{i} x_{j}\right\}_{0 \leq i<j \leq q} \cup\left\{x_{i} y_{j}-\delta_{i j} x_{i} t\right\}_{0 \leq i \leq q, 1 \leq j \leq q} \cup\left\{x_{i} z_{j}\right\}_{0 \leq i \leq q, 1 \leq j \leq k} \\
& \cup\left\{y_{i} y_{j}-\delta_{i j} y_{i} t\right\}_{1 \leq i \leq j \leq q} \cup\left\{y_{i} z_{j}\right\}_{1 \leq i \leq q, 1 \leq i \leq k} \cup\left\{z_{i} z_{j}-\delta_{i j} z_{i} t\right\}_{1 \leq i \leq j \leq k}
\end{aligned}
$$

Dabei bedeute $\delta_{i j}$ das Kronecker-Symbol.

Man rechnet leicht nach, daß die so gegebene Deformation die gewünschten Eigenschaften hat.

\section{$\S 5$. Globale projektive Kurven}

In diesem Paragraphen sei $f: \mathcal{X} \rightarrow T$ eine eigentliche, flache Familie von projektiven Kurven über einem Gebiet (= einer zusammenhängenden, offenen Teilmenge) $T \subset \mathbb{C}$, d.h. $\mathcal{X}$ ist ein abgeschlossener analytischer Unterraum von $\mathbb{P}^{n}(\mathbb{C}) \times T$ und $f$ ist die Einschränkung der Projektion nach $T$, so daß $f: \mathcal{X} \rightarrow T$ flach und $f^{-1}(t)=\mathcal{X}_{t} \times\{t\}$ für jedes $t \in T$ eine (nicht notwendig reduzierte oder reindimensionale) Kurve mit isolierten Singularitäten ist.

5.1. Für den Fall, daß alle $\mathcal{X}_{t}$ ungemischt sind, kann man aus den lokalen Aussagen von $\S 2$ entsprechende Aussagen für globale Kurven herleiten. Es gilt die folgende

Proposition. ([Gr],S.174) Ist $f: \mathcal{X} \rightarrow T$ eine eigentliche flache Familie von ungemischten projektiven Kurven über dem Gebiet $T \subset \mathbb{C}$, so gilt: 
a) Für alle $t, t^{\prime} \in T$ ist

(i) $b_{t}^{0}=b_{t^{\prime}}^{0}$,

(ii) $\mu_{t}-\mu_{t^{\prime}}=\chi_{t}-\chi_{t^{\prime}}$.

b) Folgende Bedingungen sind äquivalent:

(i) $\mu_{t}$ ist konstant,

(ii) $\delta_{t}$ und $r_{t}^{\prime}$ sind konstant,

(iii) $\chi_{t}$ ist konstant,

(iv) $b_{t}^{0}, b_{t}^{1}$ und $b_{t}^{2}$ sind konstant.

5.2. Daß Aussage 5.1a)(i) für den allgemeinen Fall nicht richtig bleiben kann, ist offensichtlich. Gültig bleibt aber (ii):

Satz. Ist $f: \mathcal{X} \rightarrow T$ eine flache Familie von projektiven Kurven wie oben, dann ist $\mu_{t}-\mu_{t^{\prime}}=\chi_{t}-\chi_{t^{\prime}}$ für alle $t, t^{\prime} \in T$.

Der Beweis ist ähnlich wie der in [Gr] für den reduzierten Fall.

5.3. Anstelle der Aussagen von Prop. 5.1b) betrachten wir:

(i) $\mu_{t}-b_{t}^{0}$ ist konstant,

(ii) $\delta_{t}$ und $r_{t}^{\prime}+b_{t}^{0}$ sind konstant,

(iii) $b_{t}^{1}-b_{t}^{2}$ ist konstant,

(iv) $b_{t}^{1}$ und $b_{t}^{2}$ sind konstant,

Offensichtlich ist dann (i) $\Leftrightarrow$ (iii) wegen Satz 5.2. Ferner gilt trivialerweise (ii) $\Rightarrow$ (i) und (iv) $\Rightarrow$ (iii). Da, falls $\delta_{t}$ konstant ist, die Fasern simultan normalisiert werden, also auch $b_{t}^{2}$ konstant ist, folgt darüberhinaus (iv) aus (ii).

Insgesamt gilt also der folgende

Satz. Ist $f: \mathcal{X} \rightarrow T$ eine flache Familie projektiver Kurven wie oben beschrieben, so gilt:

a) Folgende Aussagen sind äquivalent:

(1) $\mu_{t}-b_{t}^{0}$ ist konstant,

(2) $b_{t}^{1}-b_{t}^{2}$ ist konstant.

b) Sind $\delta_{t}$ und $r_{t}^{\prime}+b_{t}^{0}$ konstant, so auch $b_{t}^{1}$ und $b_{t}^{2}$.

Anders als im reduzierten Fall gibt es jedoch Deformationen, bei denen $b_{t}^{1}-b_{t}^{2}$ und damit $\mu_{t}-b_{t}^{0}$ konstant sind, nicht aber $b_{t}^{1}$ und $b_{t}^{2}$. Andererseits gibt es Deformationen, bei denen $b_{t}^{1}$ und $b_{t}^{2}$ konstant sind, nicht jedoch $\delta_{t}$, d.h. die Implikationen sind nicht mehr umkehrbar. 


\section{§6. Singularitäten der homologischen Dimension $\leq 2$}

In diesem Paragraphen sollen solche Singularitäten $(X, 0) \subset\left(\mathbb{C}^{m}, 0\right)$ der Codimension $\geq 1$ untersucht werden, deren lokaler Ring $\mathcal{O}_{X, 0}=\mathcal{O}_{\mathbb{C}^{m}, 0} / A$ eine $\mathcal{O}_{\mathbb{C}^{m}, 0}$-freie Auflösung der Form

$$
0 \longrightarrow \mathcal{O}_{\mathbb{C}^{m}, 0}^{n-1} \stackrel{\Psi}{\longrightarrow} \mathcal{O}_{\mathbb{C}^{m}, 0}^{n} \stackrel{\Phi}{\longrightarrow} \mathcal{O}_{\mathbb{C}^{m}, 0} \longrightarrow \mathcal{O}_{\mathbb{C}^{m}, 0} / A \longrightarrow 0
$$

hat, wo $\Psi$ eine $n \times(n-1)$-Matrix und $\Phi$ eine $1 \times n$-Matrix ist.

In diese Kategorie fallen wegen des Syzygiensatzes für reguläre lokale Ringe insbesondere alle Kurven im $\mathbb{C}^{2}$ (mit oder ohne eingebettete Komponenten), alle Hyperflächensingularitäten sowie Cohen-Macaulay-Singularitäten der Codimension 2 (vgl. [Ar], Part 1, §5). Grundlegend ist der folgende

Satz 6.1. (Hilbert-Burch) Sei $R$ ein noetherscher kommutativer Ring mit 1, $A=\left(a_{1}, \ldots, a_{n}\right) \subset R$ ein Ideal. Sei $\Phi$ die $1 \times n$-Matrix $\left(a_{1}, \ldots, a_{n}\right), \Psi$ eine $n \times(n-1)$-Matrix mit den Spalten $\Psi_{1}, \ldots, \Psi_{n-1}$ derart, daß

$$
0 \longrightarrow R^{n-1} \stackrel{\Psi}{\longrightarrow} R^{n} \stackrel{\Phi}{\longrightarrow} R \longrightarrow R / A \longrightarrow 0
$$

eine R-freie Auflösung von $R / A$ ist. Sei $\Psi^{(k)}$ die $(n-1) \times(n-1)$-Untermatrix von $\Psi$, die durch streichen der $k$-ten Zeile entsteht und $u^{(k)}:=(-1)^{n-k} \operatorname{det} \Psi^{(k)}$ der entsprechende Minor von $\Psi$. Dann gibt es einen eindeutig bestimmten Nichtnullteiler $a \in R$, so daß $a_{i}=a \cdot u^{(i)}$ für alle $i$.

Einen Beweis dieses Satzes findet man in [Bu].

Korollar. Ist $(X, 0) \subset\left(\mathbb{C}^{m}, 0\right)$ eine Singularität mit homologischer Dimension $\leq 2$ gegeben durch das Ideal $\mathrm{I}(X)=\left(g_{1}, \ldots, g_{n}\right)$, dann ist $\mathrm{I}(X)=$ $(g) \cdot\left(p_{1}, \ldots, p_{n}\right)$.

Dabei definiert $(g)$ eine Hyperfächensingularität und $\left(p_{1}, \ldots, p_{n}\right)$ eine CohenMacaulay-Singularität der Codimension 2.

6.2. Unter einer (eingebetteten) Deformation einer Singularität $(Y, 0) \subset\left(\mathbb{C}^{m}, 0\right)$ über $\left(\mathbb{C}^{k}, 0\right)$ verstehen wir einen $\operatorname{Raumkeim}(\mathcal{Y}, 0) \subset\left(\mathbb{C}^{m} \times \mathbb{C}^{k}, 0\right)$ gegeben durch ein Ideal $\mathrm{I}(\mathcal{Y}) \subset \mathcal{O}_{\mathbb{C}^{m} \times \mathbb{C}^{k}, 0}=\mathcal{O}_{m}\{\underline{t}\}, \underline{t}=\left(t_{1}, \ldots, t_{k}\right)$, so daß die Einschränkung der Projektion $f:(\mathcal{Y}, 0) \rightarrow\left(\mathbb{C}^{k}, 0\right)$ flach ist, zusammen mit einem Isomorphismus $\mathcal{O}_{Y, 0} \cong \mathcal{O}_{\mathcal{Y}, 0} \otimes_{\mathcal{O}_{\mathbb{T}^{k}, 0}} \mathbb{C}$.

Sei nun $(X, 0) \subset\left(\mathbb{C}^{m}, 0\right)$ gegeben durch $\left(g p_{1}, \ldots, g p_{n}\right)=(g) \cdot\left(p_{1}, \ldots, p_{n}\right)$, $(G, 0)$ durch $(g)$ und $(P, 0)$ durch $\left(p_{1}, \ldots, p_{n}\right)$ und sei

$$
0 \longrightarrow \mathcal{O}_{m}^{n-1} \stackrel{\left(u_{i j}\right)}{\longrightarrow} \mathcal{O}_{m}^{n} \stackrel{\left(g p_{i}\right)}{\longrightarrow} \mathcal{O}_{m} \longrightarrow \mathcal{O}_{X, 0} \longrightarrow 0
$$

eine minimale Auflösung von $\mathcal{O}_{X, 0}$. (Hier wie im folgenden bezeichne $\mathcal{O}_{m}:=$ $\left.\mathcal{O}_{\mathbb{C}^{m}, 0} \cdot\right)$ 
Sei $f_{G}:(\mathcal{G}, 0) \rightarrow\left(\mathbb{C}^{r}, 0\right)$ eine Deformation von $(G, 0)$ gegeben durch $\mathrm{I}(\mathcal{G})=$ $\left(g+\sum_{k=1}^{r} t_{k} \bar{g}_{k}\right) \subset \mathcal{O}_{m}\{\underline{t}\}$. Offensichtlich kann jede Deformation von $(G, 0)$ so dargestellt werden, und da $G$ eine Hyperfläche ist, ist jede so gegebene Funktion eine Deformation von $G$.

Sei $f_{P}:(\mathcal{P}, 0) \rightarrow\left(\mathbb{C}^{q}, 0\right)$ eine Deformation von $P$. Dann ist $\mathcal{P}$ gegeben $\operatorname{durch} \mathrm{I}(\mathcal{P})=\left(p_{i}+\sum_{k=1}^{q} s_{k} \bar{p}_{i k}\right) \subset \mathcal{O}_{m}\{\underline{s}\}$. Da $0 \longrightarrow \mathcal{O}_{m}^{n-1} \stackrel{\left(u_{i j}\right)}{\longrightarrow} \mathcal{O}_{m}^{n} \stackrel{\left(p_{i}\right)}{\longrightarrow} \mathcal{O}_{m} \longrightarrow$ $\mathcal{O}_{P, 0} \longrightarrow 0$ eine Auflösung von $\mathcal{O}_{P, 0}$ ist, ist ein derartiges Ideal genau dann eine Deformation, wenn es Funktionen $\bar{u}_{i j k} \in \mathcal{O}_{m}\{\underline{s}\}$ gibt, so daß die Sequenz

$(*): \quad 0 \longrightarrow\left(\mathcal{O}_{m}\{\underline{s}\}\right)^{n-1} \frac{\left(u_{i j}+\sum_{k=1}^{q} s_{k} \bar{u}_{i j k}\right)}{\longrightarrow}\left(\mathcal{O}_{m}\{\underline{s}\}\right)^{n}$
$\stackrel{\left(p_{i}+\sum_{k=1}^{q} s_{k} \bar{p}_{i k}\right)}{\longrightarrow} \mathcal{O}_{m}\{\underline{s}\} \longrightarrow \mathcal{O}_{\mathcal{P}, 0} \longrightarrow 0$ eine Auflösung von $\mathcal{O}_{\mathcal{P}, 0}$ ist, wenn also die Relationen-Matrix durch eine Deformation $\left(u_{i j}+\sum_{k=1}^{q} s_{k} \bar{u}_{i j k}\right)$ von $\left(u_{i j}\right)$ gegeben ist ([Ar], Theorem 5.1).

Man sieht leicht, daß in jedem Falle $f:(\mathcal{X}, 0) \rightarrow\left(\mathbb{C}^{r} \times \mathbb{C}^{q}, 0\right)$ mit $\mathcal{X} \subset$ $\left(\mathbb{C}^{m} \times \mathbb{C}^{r} \times \mathbb{C}^{q}, 0\right)$ gegeben durch $\mathrm{I}(\mathcal{X})=\mathrm{I}(\mathcal{G}) \cdot \mathrm{I}(\mathcal{P})$ eine Deformation von $(X, 0)$ ist.

6.3. Umgekehrt ist jede Deformation von $(X, 0)$ über einer glatten Basis von dieser Gestalt (Bezeichnungen von 6.2):

Proposition. Sei $(X, 0) \subset\left(\mathbb{C}^{m}, 0\right)$ eine Singularität der homologischen Dimension $\leq 2$, gegeben durch das Ideal $\left(g_{1}, \ldots, g_{n}\right)=(g)\left(p_{1}, \ldots, p_{n}\right)$. Sei $f: \mathcal{X} \rightarrow\left(\mathbb{C}^{r}, 0\right)$ eine Deformation von $(X, 0)$ gegeben durch $\mathrm{I}(\mathcal{X})=\left(g_{i}+\right.$ $\left.\sum_{k=1}^{r} t_{k} \bar{g}_{i k}\right)_{1 \leq i<n} \subset \mathcal{O}_{m}\{\underline{t}\}$. Dann gibt es für $1 \leq i \leq n, 1 \leq k \leq r$ Funktionen $\bar{g}_{k}$ und $\bar{p}_{i k}$ in $\mathcal{O}_{m}\{\underline{t}\}$, so daß gilt:

$\left(g p_{i}+\sum_{k=1}^{r} t_{k} \bar{g}_{i k}\right)_{1 \leq i \leq n}=\left(g+\sum_{k=1}^{r} t_{k} \bar{g}_{k}\right)\left(p_{i}+\sum_{k=1}^{r} t_{k} \bar{p}_{i k}\right)_{1 \leq i \leq n}$, wobei $f_{G}: \mathcal{G} \rightarrow\left(\mathbb{C}^{r}, 0\right)$ mit $\mathrm{I}(\mathcal{G})=\left(g+\sum_{k=1}^{r} t_{k} \bar{g}_{k}\right)$ eine Deformation von $(G, 0)$ sowie $f_{P}: \mathcal{P} \rightarrow\left(\mathbb{C}^{r}, 0\right)$ mit $\mathrm{I}(\mathcal{P})=\left(p_{i}+\sum_{k=1}^{r} t_{k} \bar{p}_{i k}\right)_{1 \leq i \leq n}$ eine Deformation von $(P, 0)$ ist.

Beweis: $\quad$ Es ist $0 \longrightarrow \mathcal{O}_{m}^{n-1} \stackrel{\left(u_{i j}\right)}{\longrightarrow} \mathcal{O}_{m}^{n} \stackrel{\left(g p_{i}\right)}{\longrightarrow} \mathcal{O}_{m} \longrightarrow \mathcal{O}_{X, 0} \longrightarrow 0$ eine Auflösung von $\mathcal{O}_{X, 0}$.

Da $f$ eine Deformation ist, gibt es $\bar{u}_{i j k}$, so daß $(*)$ eine Auflösung von $\mathcal{O}_{\mathcal{X}, 0}$ ist. Nach Hilbert-Burch gibt es dann $\tilde{g}$ und $\tilde{p}_{i}$ in $\mathcal{O}_{m}\{\underline{t}\}$, so daß $g p_{i}+\sum_{k=1}^{q} t_{k} \bar{g}_{i k}=$ $\tilde{g} \tilde{p}_{i}$.

Dann ist $g p_{i} \equiv \tilde{g} \tilde{p}_{i} \bmod \underline{t}=(\tilde{g} \bmod \underline{t})\left(\tilde{p}_{i} \bmod \underline{t}\right)$, und daher $g \equiv \tilde{g} \bmod \underline{t}$ und $p_{i} \equiv \tilde{p}_{i} \bmod \underline{t}\left(\mathrm{Da} V(g)\right.$ ebenso wie $V(\tilde{g} \bmod \underline{t})$ die Codimension $1, V\left(p_{1}, \cdots, p_{n}\right)$ und $V\left(\tilde{p}_{1} \bmod \underline{t}, \cdots, \tilde{p}_{n} \bmod \underline{t}\right)$ aber die Codimension 2 haben). Deshalb gibt es $\bar{g}_{k}, \bar{p}_{i k}$, so daß $\tilde{g}=g+\sum_{k=1}^{r} t_{k} \bar{g}_{k}$ und $\tilde{p}_{i}=p_{i}+\sum_{k=1}^{r} t_{k} \bar{p}_{i k}$.

Da $(G, 0)$ eine Hyperflächensingularität ist, ist die durch $\tilde{g}$ definierte Funktion $f_{G}$ eine Deformation von $(G, 0)$.

Da $(*)$ eine Auflösung von $\mathcal{O}_{\mathcal{P}, 0}$ ist, ist die durch die $\tilde{p}_{i}$ definierte Funktion $f_{P}$ eine Deformation von $(P, 0)$. 
Unter Berücksichtigung der Tatsache, daß die semiuniverselle Deformation einer isolierten Cohen-Macaulay-Singularität der Codimension 2 ebenso wie die einer Hyperfläche eine glatte Basis hat ([Ar]), ergibt sich

Korollar. Sei $(X, 0) \subset\left(\mathbb{C}^{m}, 0\right)$ eine Singularität der homologischen Dimension $\leq 2$ gegeben durch $(g)\left(p_{1}, \ldots, p_{n}\right)$ und sei $(G, 0)$ durch $(g)$ und $(P, 0)$ durch $\left(p_{1}, \ldots, p_{n}\right)$ gegeben. Dann hat die semiuniverselle Deformation von $(X, 0)$ die Gestalt $f:(\mathcal{X}, 0) \rightarrow\left(\mathbb{C}^{r} \times \mathbb{C}^{q}, 0\right),(\mathcal{X}, 0) \subset\left(\mathbb{C}^{m} \times \mathbb{C}^{r} \times \mathbb{C}^{q}, 0\right)$ gegeben durch $\mathrm{I}(\mathcal{X})=\left(g+\sum_{k=1}^{r} t_{k} \bar{g}_{k}\right)\left(p_{i}+\sum_{k=1}^{q} s_{k} \bar{p}_{i k}\right)=\mathrm{I}(\mathcal{G}) \cdot \mathrm{I}(\mathcal{P})$, wobei $\mathrm{I}(\mathcal{G})$ bzw. $\mathrm{I}(\mathcal{P})$ die semiuniversellen Deformationen von $(G, 0)$ bzw. $(P, 0)$ repräsentieren.

Beweis: Nach 6.2 ist $f$ eine Deformation von $(X, 0)$ und die semiuniverselle Deformation hat eine Basis der Dimension $\geq r+q$. Nach 6.3 ist $f$ aber versell und damit semiuniversell.

Bemerkung. Ist $(X, 0) \subset\left(\mathbb{C}^{2}, 0\right)$ eine isolierte Kurvensingilarität mit eingebetteter Komponente, dann definiert die eingebettete Komponente einen dicken Punkt $P$ der Länge $\varepsilon_{0}=\varepsilon(X, 0)$. Nach dem Korollar erhalten wir alle Deformationen von $(X, 0)$ durch Vereinigung beliebiger Deformationen von $\left(X^{\text {red }}, 0\right)$ und von $P$. Speziell gilt für die allgemeine Faser einer beliebigen Deformation von $(X, 0): b^{0}\left(\left(\mathcal{X}_{t}\right)^{1}\right)=1,1 \leq b^{0}\left(\left(\mathcal{X}_{t}\right)^{0}\right) \leq \varepsilon_{0}$, und jeder Wert wird bei einer geeigneten Deformation angenommen. Es ist stets $\varepsilon_{t}=\varepsilon_{0}, X$ besitzt keine reine Glättung (außer $X$ ist reduziert) und für jede Glättung gilt: $b^{0}\left(\mathcal{X}_{t}\right)=b^{0}\left(\left(\mathcal{X}_{t}\right)^{0}\right)+1=\varepsilon_{0}+1, b^{1}\left(\mathcal{X}_{t}\right)=\mu\left(X_{\text {red }}, 0\right)=\mu(X, 0)+2 \varepsilon_{0}$.

\section{Literatur}

[Ar] : Artin, M.: Deformations of Singularities, Tata Institute of Fundamental Research, Bombay 1976

[B-G] : Buchweitz, R.-O. u. Greuel, G.-M.: The Milnor-number and deformations of complex curve singularities, Inv. math. 58(1980), 241-281

[Bu] : Burch, L.: On ideals of finite homological dimension in local rings, Proc. Cam. Phil. Soc. 64(1968), 941-948

[Fi] : Fischer, G.: Complex analytic geometry, Lecture Notes in Math. 538, Springer Verlag, Berlin, Heidelberg, New York 1976

[Gr] : Greuel, G.-M.: On deformations of curves and a formula of Deligne, in: Aroca-Buchweitz-Giusti-Merle Hrsg.: Algebraic Geometry, Lecture Notes in Math. 961, Springer Verlag, Berlin, Heidelberg, New York 1982

[Ma] : Matsumura, H.: Commutative Algebra (second edition), Benjamin/Cummings, Reading, Massachusetts 1980

[St] : Steenbrink, J.: Mixed Hodge-structures and Singularities, Manuskript

[Te] : Teissier, B.: Résolution simultanée I,II, in: Séminaire sur les Singularités des Surfaces. Palaisau 1976-77. Ed. M. Demazure, H. Pinkham, B. Teissier, Lecture Notes in Math. 777, Springer Verlag, Berlin, Heidelberg, New York 1980 
Christian Brücker

Fachbereich Mathematik und Informatik

Fernuniversität Hagen 5800 Hagen
Gert-Martin Greuel

Fachbereich Mathematik

Universität Kaiserslautern

6750 Kaiserslautern

This article was processed by the author using the Springer-Verlag TEX mamath macro package 1990. 